\title{
A new technique for determining orientation and motion of a 2-D, non-planar magnetopause
}

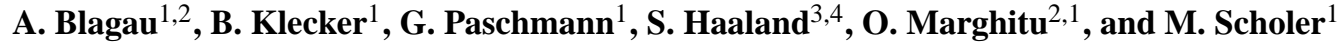 \\ ${ }^{1}$ Max-Planck-Institute for extraterrestrial Physics, Garching, Germany \\ ${ }^{2}$ Institute for Space Sciences, Bucharest, Romania \\ ${ }^{3}$ Max-Planck Institute for Solar System Research, Katlenburg-Lindau, Germany \\ ${ }^{4}$ Department of Physics, University of Bergen, Norway
}

Received: 29 December 2008 - Revised: 4 March 2010 - Accepted: 8 March 2010 - Published: 16 March 2010

\begin{abstract}
For a four-point mission like Cluster, the differences in position and time when the satellites detect the magnetopause or any other discontinuity, can be used to infer the discontinuity local orientation, thickness and motion. This timing technique, commonly assuming a planar geometry, offers an independent check for various single-spacecraft techniques.

In the present paper we propose an extension of the timing method, capable of determining in a self-consistent way the macroscopic parameters of a two-dimensional, non-planar discontinuity. Such a configuration can be produced by a local bulge or indentation in the magnetopause, or by a large amplitude wave traveling on this surface, and is recognized in Cluster data when the single spacecraft techniques provide different individual normals contained roughly in the same plane. The model we adopted for the magnetopause assumes a layer of constant thickness of either cylindrical or parabolic shape, which has one or two degrees of freedom for the motion in the plane of the individual normals. The method was further improved by incorporating in a selfconsistent way the requirement of minimum magnetic field variance along the magnetopause normal. An additional assumption, required in a previously proposed non-planar technique, i.e. that the non-planarity has negligible effects on the minimum variance analysis, is thus avoided.

We applied the method to a magnetopause transition for which the various planar techniques provided inconsistent results. By contrast, the solutions obtained from the different implementations of the new 2-D method were consistent and stable, indicating a convex shape for the magne-
\end{abstract}

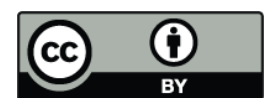

Correspondence to: A. Blagau

(blagau@spacescience.ro) topause. These solutions perform better than the planar solutions from the normal magnetic field variance perspective. The magnetopause dynamics and the presence of a non-zero normal magnetic field component in the analyzed event are discussed.

Keywords. Magnetospheric physics (Magnetopause, cusp, and boundary layers; Instruments and techniques) - Space plasma physics (Discontinuities)

\section{Introduction}

The macroscopic parameters that characterize a plasma discontinuity like the terrestrial magnetopause (MP), i.e. the orientation, motion, and thickness, are rapidly changing and therefore need to be determined for each particular event.

The motivation of finding the MP macroscopic parameters arises for a number of reasons. One is the problem of studying the large-scale dynamical processes that take place at this interface, like for example large amplitude surface waves. For that purpose, only a moderately accurate knowledge about the MP normal direction and motion is sufficient. Another important question is whether the magnetosphere and the magnetosheath regions are magnetically connected, i.e. whether a non-zero average normal magnetic field component $\left\langle B_{n}\right\rangle$ is present or not at the MP. Since, even when the MP behaves like a rotational discontinuity, $\left\langle B_{n}\right\rangle$ at this interface is very small (typically below $10 \%$ of the total magnetic intensity), an accurate determination of the normal direction is needed to answer that question. The same requirement applies when establishing a plasma flow across the MP. Then, the knowledge of the MP thickness allows us to estimate the spatial length-scale of the processes occurring in

Published by Copernicus Publications on behalf of the European Geosciences Union. 
that region, providing an indication on the plasma regime whether MHD or kinetic. It also allows us to compute, via Ampère's law, the MP current density, an important parameter in the study of plasma instabilities.

The earliest methods for determining the MP orientation use data collected by a single satellite and rely on certain simplifying assumptions regarding the discontinuity, like planar geometry, time-stationarity, and variations only along the normal direction for all physical quantities. The first such technique, the minimum variance analysis of the magnetic field (MVAB), developed by Sonnerup and Cahill (1967), is based on $\nabla \cdot \boldsymbol{B}=0$. Under the mentioned assumptions, this law implies a constant magnetic field component along the discontinuity normal. Consequently, using satellite measurements taken during the traversal, the MVAB algorithm identifies the direction along which the magnetic variance is minimized and associates it with the MP normal.

In some cases one can combine results from MVAB and from the so-called deHoffmann-Teller (HT) analysis (e.g., Khrabrov and Sonnerup, 1998a; Paschmann and Sonnerup, 2008), to find also the MP normal velocity. In the HT analysis, one searches for a specific reference frame where plasma flow becomes aligned with the magnetic field and thus the motional electric field vanishes. If, based on MP data, the HT frame is identified, one can interpret the measurements as caused by a time-stationary structure moving over the point of observation. Then, by projecting the velocity of the HT frame along the MP normal, one obtains the MP normal velocity.

Another single-spacecraft method, the Minimum Faraday Residue (MFR), was developed based on magnetic flux conservation across the discontinuity (Khrabrov and Sonnerup, 1998b). Here the normal direction and the normal velocity (assumed constant) are found by minimizing the Faraday residue, i.e. the variance of the tangential electric field component in the discontinuity frame. More recently various other single-spacecraft techniques were proposed, each based on the conservation of some particular physical quantity across the discontinuity. A detailed and unified description of these techniques is provided in Sonnerup et al. (2006).

For a four-spacecraft mission like Cluster, additional methods for determining the orientation and motion of a discontinuity are available. These are the timing methods, that use the differences between the positions and times of the MP encounter by the satellites. In general, the timing methods assume also a planar geometry and a steady normal orientation. The first such technique, called time of arrival method, was proposed by Russell et al. (1983), and in addition assumes a constant velocity for the discontinuity during the entire event. Haaland et al. (2004) introduced a four-point, planar timing technique that assumes a constant thickness for the discontinuity at the scale of the inter-spacecraft separation distance, allowing thus for a variable normal velocity. In that paper, arguments in favor of a constant MP thickness as opposed to those in favor of a constant velocity, are provided. One such argument is that, due to its low mass per unit cross-section, the MP will experience high accelerations even for small pressure imbalances, while, on the other hand, the compression is relatively small (of the order of a few percent). Experimental evidence (Dunlop et al., 2001, 2002) supports the idea of a MP undergoing rapid changes in the normal velocity over time-periods comparable to the crossing duration, with relative small variation of its thickness. A detailed description of these two approaches, termed Constant Velocity Approach (CVA) and Constant Thickness Approach (CTA), can be found in Haaland et al. (2004).

The problem of determining the macroscopic parameters of a more realistic, non-planar discontinuity, when four points of measurements are available, was investigated by Mottez and Chanteur (1994) and Dunlop and Woodward $(1998,1999)$. These authors developed a method which, in the first stage, applies the MVAB technique on each satellite to determine the local discontinuity normal, and afterwards combines these results with the information about the position and time of discontinuity encounter by each satellite. For a 3-D discontinuity, with a regular geometry at the scale of the inter-spacecraft separation distance, the two principal radii of curvature that characterize the surface as well as the velocity and acceleration of the structure can thus be obtained. An important assumption of this method is that the individual normals obtained from a planar technique, i.e. MVAB, are not significantly affected by the local curvature and motion of the non-planar discontinuity during the satellite traversal. To our knowledge, the technique has not been quantitatively applied to a four-spacecraft crossing event.

In the present paper we developed a method for determining the macroscopic parameters of a two-dimensional (2-D), non-planar discontinuity, assuming that its thickness can be considered as constant. With the new method the discontinuity local orientation, motion, thickness and curvature are obtained simultaneously in a self-consistent way, removing thus the need of an additional assumption, required in the above mentioned non-planar technique, i.e. that of negligible effects due to non-planarity on the results provided by MVAB. In the first instance we extend the planar CTA timing technique, by proposing two simple geometries for the discontinuity layer, i.e. that of either a cylindrical or a parabolic shape. Later on, the method is improved by incorporating the requirement of minimum magnetic field variance along the instantaneous, local normal, therefore relying simultaneously on the timing information and on the $\nabla \cdot \boldsymbol{B}=0$ law. The new method was developed to address the needs described at the beginning of this section, when the MP non-planarity adversely influences the values of the macroscopic parameters provided by the planar techniques.

We will illustrate our technique with a particular MP crossing by Cluster, when the boundary acquired a 2-D shape, possibly due to a large amplitude surface wave produced by the Kelvin-Helmholtz mechanism. A detailed comparison between the results provided by the planar timing analysis 
and by the single-spacecraft techniques of MVAB, MFR, and HT, on one hand, and those provided by the 2-D method, on the other hand, will show the advantage of the latter. With the new technique, the MP dynamics and the presence of a non-zero normal magnetic field component in this particular event, will be discussed.

The paper is organized as follows: Sect. 2 introduces the MP crossing from 24 June 2003, providing evidence that the discontinuity has a 2-D, non-planar geometry in this event. Section 3 describes the principles of the new method, with additional details given in Appendix A. Section 4 briefly discusses how the MP moved on 24 June 2003, based on three solutions obtained by applying the 2-D method. An in-depth comparison between one particular 2-D solution and the results provided by the standard, planar methods is presented in Sect. 5, with additional details given in Appendix B. The parameters that characterize all the valid 2-D solutions and the corresponding error analysis are presented in Sect. 6 and in Appendix C. Section 7 provides a discussion of the results obtained with the 2-D method. Section 8 summarizes the results and provides a general perspective, including a list of topics to be addressed in the future.

\section{A case of a 2-D, non-planar magnetopause}

In this section we present a MP crossing by Cluster on 24 June 2003, to be used as demonstration case for the new 2-D, non-planar technique. We will show that single and multisatellite planar methods yield inconsistent results, and will provide arguments that the MP has actually a 2-D, non-planar geometry in this case. The direction along which the MP shape does not change, called hereafter the invariant direction, and the timing information, both used as input parameters in the new technique, will be determined from the data.

In the present paper we use data from three experiments on-board Cluster. The flux-gate magnetometer (FGM) provides the DC magnetic field vector with an accuracy of about $0.1 \mathrm{nT}$ and a time-resolution of up to 67 samples per second (see Balogh et al., 2001). The Electric Field and Wave (EFW) experiment provides the two electric field components in the spin plane at 25 samples/s in normal mode (see Gustafsson et al., 2001). In favorable conditions the component along the spin axis can be inferred under the assumption that $\boldsymbol{E} \cdot \boldsymbol{B}=0$, provided that the magnetic field vector is not too close to the spin-plane. The Cluster Ion Spectrometry (CIS) experiment measures the three-dimensional velocity distribution function of the ions. Based on that, the plasma moments (density, bulk velocity vector, pressure tensor, and heat flux vector) are computed and transmitted to the ground every spacecraft spin period, i.e. every $4 \mathrm{~s}$ (see Rème et al., 2001).

Figure 1 presents the Cluster orbit and configuration for the 24 June 2003 event. The transition that will be analyzed is an inbound crossing and occurred around 07:37:00 UT at the MP dawn flank, near $[-7.7,-17.3,-4]$ Earth radii $\left(R_{\mathrm{E}}\right)$
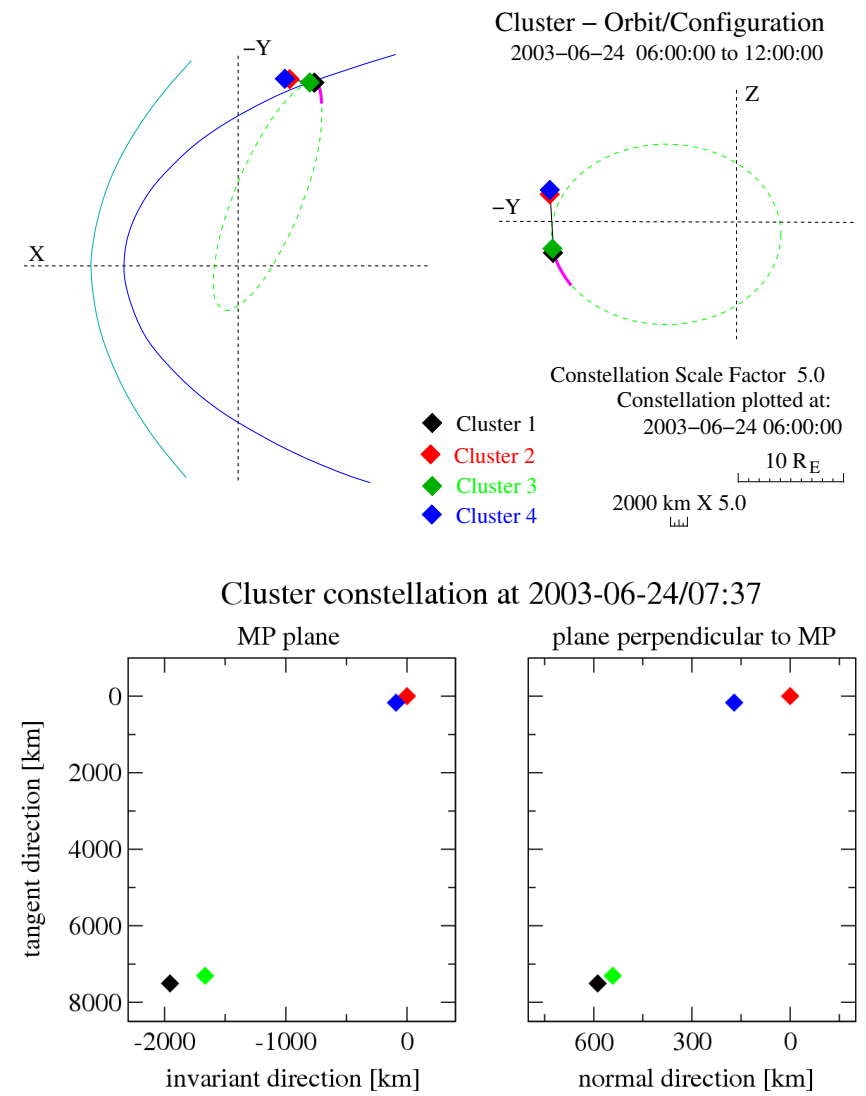

Fig. 1. Upper part: Cluster orbit on 24 June 2003 projected on the $\mathrm{XY}$ and ZY planes of the GSE reference system. The distances between satellites, shown in the mission color code, are increased by a factor of 5. Lower part: Projections of Cluster configuration on the MP plane (left) and on a plane containing the MP normal (right), at the time of the event. More details are given in the text.

in GSE coordinates. In the upper part of the figure, showing the orbit (adapted from Cluster Science Data System web page), a model MP and bow-shock are drawn and the distance between the satellites is scaled by a factor of 5 . For this event, the four satellites are traveling in pairs, with Cluster 1 (C1) close to $\mathrm{C} 3$ (at $\sim 350 \mathrm{~km}$ ) and $\mathrm{C} 2$ close to $\mathrm{C} 4$ (at $\sim 250 \mathrm{~km}$ ). The distance between the pairs is $\sim 7500 \mathrm{~km}$.

On the bottom part of Fig. 1, two plane projections of the Cluster configuration at the time of traversal are shown, both of them with $\mathrm{C} 2$ - the first satellite entering the MP - at the origin. On the left we show the projection on the "MP plane", defined as the plane perpendicular to the direction resulting from averaging the individual normals, i.e. the normals obtained from applying MVAB at each satellite (details will be given in Sect. 2.1). On the right projection, the $\boldsymbol{x}$ axis is chosen to be along this average MVAB normal direction. As one can clearly see from the latter, the expected sequence of the MP crossings is $\mathrm{C} 2, \mathrm{C} 4, \mathrm{C} 3$ and $\mathrm{C} 1$. 


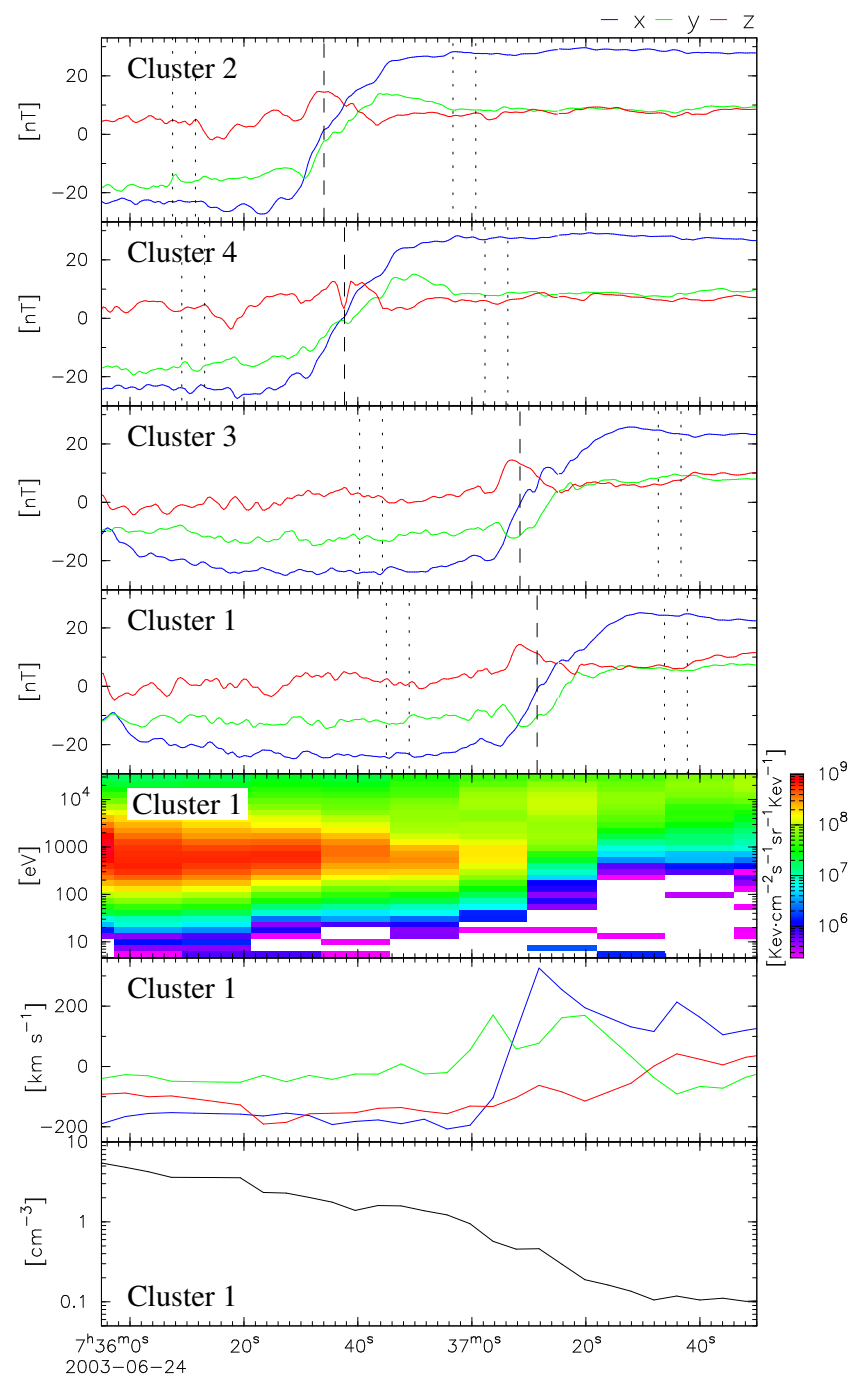

Fig. 2. First four panels: Magnetic field in GSE reference frame measured by Cluster during the MP crossing on 24 June 2003. The panel order, i.e. $\mathrm{C} 2, \mathrm{C} 4, \mathrm{C} 3$, and $\mathrm{C} 1$, corresponds to the sequence of MP encounter. The $\mathrm{x}, \mathrm{y}$, and $\mathrm{z}$ components are indicated by the blue, green and red lines, respectively. The minimum and maximum length intervals entering the MVAB nested analysis (vertical dotted lines) together with their common central time (vertical dashed lines) are shown for each satellite. Last three panels: Ions differential energy spectrogram, bulk velocity components in GSE, and density, as measured by the CIS/Hot Ion Analyser on-board C1.

The same sequence is indeed seen in Fig. 2, which presents in the first four panels the magnetic field components measured by each spacecraft in the GSE reference frame. The significance of the various vertical lines indicated in these panels is explained in Sect. 2.1. From the last three panels, showing the ion data, i.e. the differential energy spectrogram, the bulk velocity components in GSE, and the density, measured by the CIS/Hot Ion Analyser on-board C1, one can clearly notice the inbound character of the transition.
The Cluster configuration on 24 June 2003, not being a tetrahedron, may raise concerns about the accuracy of the results obtained with a timing based method. The errors in determining the MP parameters with the new method will be thoroughly analyzed in Appendix C, where it will be shown they stay at an acceptable level. On that point, Fig. 2 illustrates one important aspect of the demonstration case, namely a "clean" MP transition at each satellite, with well defined asymptotic levels and a smooth magnetic field transition. This ensures a reliable determination of the timing information (see Sect. 2.3), which indicates clearly separated satellite transitions, that are at least $2.75 \mathrm{~s}$ apart (see Table 2 in that section).

\subsection{The results of MVAB - finding the invariant direction $l$}

In order to reduce the influence of small-scale fluctuations on the MVAB results, we "boxcar-averaged" the initial $0.2 \mathrm{~s}$ magnetic data by using a boxcar of $1 \mathrm{~s}$ width, followed by a resampling to $0.4 \mathrm{~s}$ time resolution. Next, for each satellite, we chose eleven symmetric, nested intervals, centred on a point near the middle of the structure (the central times, the minimum and maximum length of the intervals are presented in Fig. 2). For each of these eleven intervals the MVAB was performed, first with the constraint $\langle\boldsymbol{B}\rangle \cdot \boldsymbol{n}=0$, where $\langle\boldsymbol{B}\rangle$ is the average magnetic field (see for example Sonnerup and Scheible, 1998), and, second, without this constraint. In each variant of the method, the average direction, obtained as the component by component average of the eleven normals, was computed.

The results are presented in the left and central part of Table 1. There, for each satellite, the GSE components of the individual normal vectors provided by both MVAB versions are shown, including the ratio $\lambda_{1} / \lambda_{2}$, between the magnetic field variance along the directions of maximum and intermediate variance (constrained analysis), and the ratio $\lambda_{2} / \lambda_{3}$, between the magnetic field variance along the directions of intermediate and minimum variance (unconstrained analysis). In addition, for the unconstrained MVAB, the average normal magnetic field component $\left\langle B_{n}\right\rangle=\langle\boldsymbol{B}\rangle \cdot \boldsymbol{n}$ is indicated. The ratio of eigenvalues provides a criterion for judging the reliability of the normal determination (see Sonnerup and Scheible, 1998). A rule of thumb requires a value of this ratio $\gtrsim 20$ for the constrained MVAB method and values $\gtrsim 10$ for the unconstrained method. As seen from Table 1, the criterion is easily fulfilled by the constrained MVAB normals, but not by the unconstrained MVAB normals. Another fact that makes the unconstrained case suspicious is the angle of $\sim 11^{\circ}$ between the C2 and C4 individual normals, which is unreasonably large considering that the satellites are crossing the MP almost simultaneously at a small distance.

Based on these arguments, we shall use only the results provided by the constrained $(\langle\boldsymbol{B}\rangle \cdot \boldsymbol{n}=0)$, MVAB technique. The analysis also indicates a fairly time-stationary MP; for 
Table 1. Results from the constrained (to $\left\langle B_{n}\right\rangle=0$ ) and unconstrained MVAB averaged over the eleven nested intervals, as well as from the constrained (to $\left\langle B_{n}\right\rangle=0$ ) MFR technique. For each satellite, ordered here by the time of MP encounter, the GSE components of the normal vector, together with the corresponding eigenvalues ratio are presented. In case of the unconstrained MVAB, the resulting average normal magnetic field component is provided as well. The last column shows the angle between the MFR normals and the plane of the normals. More details are provided in the text.

\begin{tabular}{|c|c|c|c|c|c|c|c|c|c|c|c|c|c|c|}
\hline & \multicolumn{4}{|c|}{ Constrained MVAB } & \multicolumn{5}{|c|}{ Unconstrained MVAB } & \multicolumn{5}{|c|}{ Constrained MFR } \\
\hline & $n_{x}$ & $n_{y}$ & $n_{z}$ & $\lambda_{1} / \lambda_{2}$ & $n_{x}$ & $n_{y}$ & $n_{z}$ & $\lambda_{2} / \lambda_{3}$ & $\left\langle B_{n}\right\rangle[n T]$ & $n_{x}$ & $n_{y}$ & $n_{z}$ & $\lambda_{1} / \lambda_{2}$ & $\Omega[\mathrm{deg}]$ \\
\hline $\mathrm{C} 2$ & 0.465 & -0.842 & -0.272 & 152 & 0.467 & -0.851 & -0.240 & 3.2 & 0.22 & 0.573 & -0.807 & -0.145 & 11.7 & 3.3 \\
\hline $\mathrm{C} 4$ & 0.447 & -0.851 & -0.274 & 135 & 0.458 & -0.887 & -0.058 & 2.5 & 1.34 & 0.581 & -0.807 & -0.106 & 2.9 & 2.8 \\
\hline $\mathrm{C} 3$ & 0.357 & -0.818 & -0.451 & 166 & 0.331 & -0.714 & -0.618 & 7.7 & -1.18 & 0.417 & -0.778 & -0.470 & 2.0 & 4.9 \\
\hline $\mathrm{C} 1$ & 0.333 & -0.827 & -0.454 & 138 & 0.305 & -0.716 & -0.627 & 6.2 & -1.42 & 0.361 & -0.823 & -0.439 & 2.8 & 0.4 \\
\hline
\end{tabular}

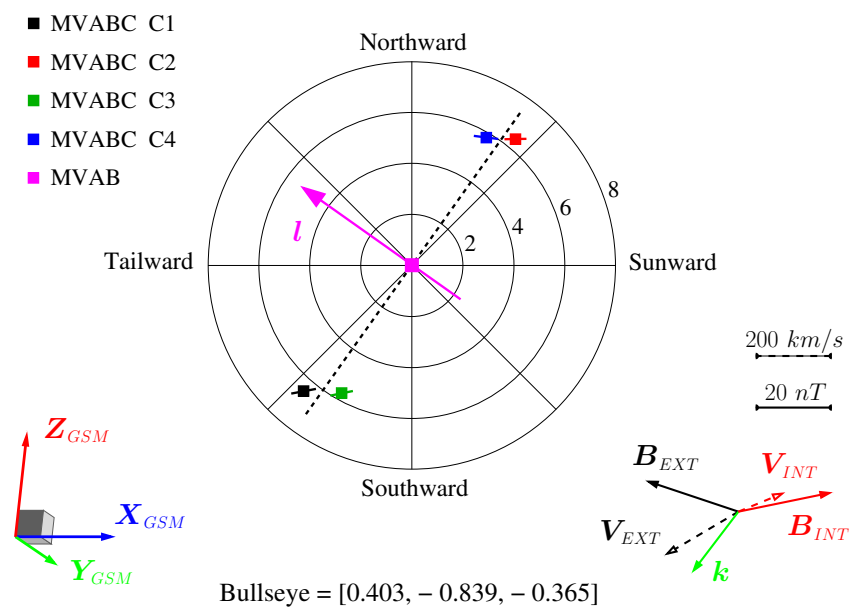

Fig. 3. Polar plot showing the orientation of the four individual normals provided by the constrained MVAB technique (the squares in the mission color code) with respect to their average orientation, taken as reference direction (the central square in magenta). The circles designate directions of equal inclination, in degrees. The individual normals are located roughly in one plane (dashed line), indicating a two-dimensional MP with the invariant direction oriented perpendicular (the unit vector $\boldsymbol{l}$ in magenta). In the lower left corner, the orientation of GSM coordinate system is shown, as seen when looking against the reference, $\langle\boldsymbol{n}\rangle_{\text {MVAB }}$ direction. The lower right corner presents the tangential components of the magnetic field and velocity flow, as measured by $\mathrm{C} 1$, in the regions adjacent to the MP interval: in the magnetosphere (red) and in the magnetosheath (black). The scales of the vectors are also shown. The unit vector $\boldsymbol{k}$ (green) is in the tangent plane and perpendicular to $\boldsymbol{l}$. More details are provided in the text.

example, in case of $\mathrm{C} 3$ the eleven normals corresponding to the nested intervals are less than $1^{\circ}$ apart from their average.

Figure 3 is a polar plot showing the orientation of the four individual normals obtained from constrained MVAB (the colored squares, symbol MVABC) with respect to their av-

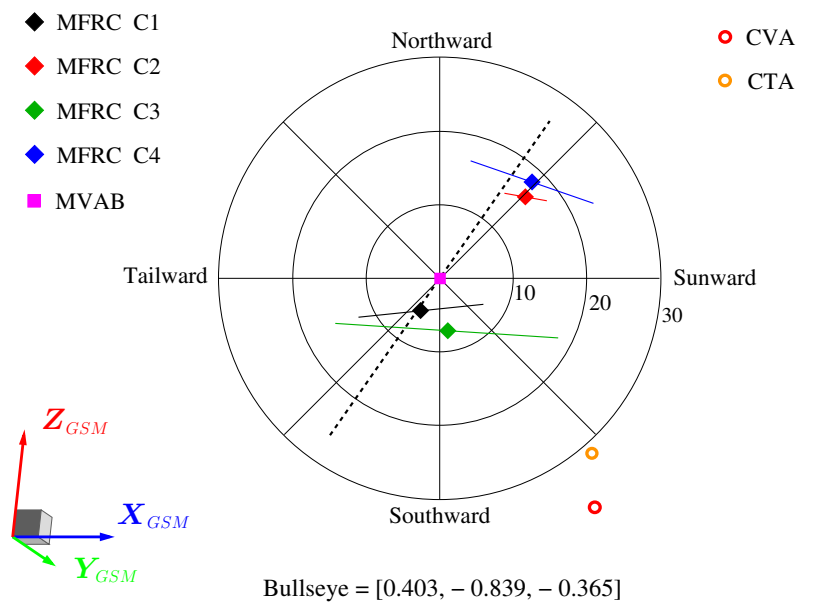

Fig. 4. Polar plot having the same reference direction as Fig. 3 for an increased angular range. It shows the orientation of the individual normals provided by the constrained MFR technique (rhombs), the CVA (red open circle), and CTA (golden open circle) normals, provided by the planar timing analysis. The plane of the normals (see text) is indicated with a dashed line.

erage, $\langle\boldsymbol{n}\rangle_{\text {MVAB }}$, taken as reference direction (the magenta square at the center, symbol MVAB). The vertical line is oriented along the $\langle\boldsymbol{n}\rangle_{\mathrm{MVAB}} \times \boldsymbol{X}_{\mathrm{GSE}}$ direction (roughly along the south-north direction) and the horizontal line completes the cartesian frame. The components of $\langle\boldsymbol{n}\rangle_{\text {MVAB }}$ in the GSE reference frame are indicated at the bottom. Its orientation with respect to the GSM reference frame can be judged by looking at the unit vectors on the bottom left part of the figure. The circles designate directions of equal inclination with respect to $\langle\boldsymbol{n}\rangle_{\text {MVAB }}$, in degrees. For each individual normal, an error bar is drawn, indicating the orientation uncertainty due to the statistical errors (see Sonnerup and Scheible, 1998).

Figure 3 shows that the individual normals are roughly contained in one plane. With the dashed line we show the 
intersection with the closest (in the least square sense) plane situated between the normals. All four MVAB normals lie within $0.7^{\circ}$ of it. Between the normals detected by $\mathrm{C} 2$ and $\mathrm{C} 1$ (i.e. the first and the last satellite crossing the MP) the difference in orientation is about $12.5^{\circ}$. Considering that the individual, constrained MVAB normals appear to be very reliable, we interpret the results as the effect of Cluster encountering a 2-D, non-planar MP. The plane indicated by the dashed line will be called hereafter the plane of the normals. The invariant direction, used as input parameter in the new 2$\mathrm{D}$ technique, is perpendicular to that plane and indicated by the unit vector $\boldsymbol{l}$. Its components in the GSE reference frame are $[-0.748,-0.532,0.398]$.

The individual normals are grouped two by two $(\mathrm{C} 1$ with C3 and C2 with C4), a consequence of the Cluster satellites crossing the MP in pairs. Due to this particular formation, one cannot rule out a priori the possibility of a planar MP that changed its orientation between the crossing of the two pairs. However, one can consider such a situation a limiting case, when the 2-D MP surface has a very large radius of curvature. It will become clear in Sect. 3 that solutions of this kind are not excluded by the new method. For the 24 June 2003 event, we shall come back to this aspect in Sect. 5, where the results obtained by applying the new 2-D method are discussed.

In the lower right corner of Fig. 3 we plotted the tangential components of the magnetic field and flow velocity vectors measured by $\mathrm{C} 1$ in the regions adjacent to the MP, i.e. the magnetosphere interior (in red, the vectors $\boldsymbol{B}_{\mathrm{INT}}$ and $\boldsymbol{V}_{\text {INT }}$ ) and the magnetosheath (in black, the vectors $\boldsymbol{B}_{\mathrm{EXT}}$ and $\boldsymbol{V}_{\text {EXT }}$ ). Each vector is an average over 5 data points, corresponding to the time intervals [07:37:36-07:37:52], and [07:36:31-07:36:47] respectively. The angle between the two flow directions is $\sim 172^{\circ}$ and the magnetic field shear angle is $\sim 150^{\circ}$.

At the bottom of Fig. 1, showing the Cluster constellation at the time of the event, the $\boldsymbol{x}$ axis is oriented along $\boldsymbol{l}$ in the left projection, while in the right projection it lies along $\langle\boldsymbol{n}\rangle_{\text {MVAB }}$. The $\boldsymbol{y}$ axis, the same in both pictures, is oriented along $\langle\boldsymbol{n}\rangle_{\text {MVAB }} \times \boldsymbol{l}$.

Additional arguments in favor of a 2-D geometry for the MP on 24 June 2003 will be provided in Sects. 2.2 and 2.4, where the results obtained by applying the planar MFR and timing analysis methods will be shown.

\subsection{The results of MFR}

For carrying out the MFR analysis we need both magnetic field and high-resolution electric field data ${ }^{1}$. Unfortunately, during some parts of the crossings, the magnetic field vector was close to the spacecraft spin plane. Because this leads to

\footnotetext{
${ }^{1}$ The relatively fast transitions seen by Cluster on 24 June 2003, results in a number of data points too low to apply MFR to the $4 \mathrm{~s}$, spin-resolution convection electric field, $\boldsymbol{E}=-\boldsymbol{v} \times \boldsymbol{B}$, derived from the plasma velocity $v$ measured by CIS.
}

high uncertainties when constructing the third, spin-aligned electric field component, for those parts of the MP transition the data cannot be used. However, for all the Cluster satellites, the interval used in the MFR analysis is wide enough to include the MP central portion (i.e. corresponding to the central interval $\left[T_{c i}-\tau_{i}, T_{c i}+\tau_{i}\right]$ defined in Sect. 2.3).

Like in the MVAB case, to reduce the influence of smallscale fluctuations on the MFR results, we "boxcar-averaged" the high-resolution data using a $2 \mathrm{~s}$ boxcar window, followed by a resampling to $0.5 \mathrm{~s}$ time-resolution. Each individual MFR normal was obtained by constraining the average normal magnetic field component to zero, i.e. $\langle\boldsymbol{B}\rangle \cdot \boldsymbol{n}=0$. The results are presented in the right part of Table 1 . For each satellite, the GSE components of the normal vectors, together with the eigenvalues ratio $\lambda_{1} / \lambda_{2}$ are shown. The last column presents the angle $\Omega$, made by the individual MFR normals with respect to the plane of the normals, determined in Sect. 2.1.

As Table 1 shows, the MFR normals are not so well defined, except, perhaps, the $\mathrm{C} 2$ normal, with a ratio $\lambda_{1} / \lambda_{2}$ around 11.7. One possible explanation could be that, due to the already mentioned data gaps, the intervals used in the MFR analysis do not include the whole MP transition. Indeed, in case of $\mathrm{C} 2$, approx. $93.7 \%$ of the magnetic field rotation occurs during the MFR interval (the largest fractional change) whereas for $\mathrm{C} 3$, when $\lambda_{1} / \lambda_{2} \simeq 2$, the MFR interval covers only $76.2 \%$ of the total magnetic field change (the smallest fractional change).

Figure 4 is a polar plot with the same reference direction as Fig. 3, i.e. $\langle\boldsymbol{n}\rangle_{\text {MVAB }}$ (central magenta square), but has an increased angular range. The plane of the normals, obtained in Sect. 2.1, is indicated by the dashed line. The individual MFR normals are shown as colored rhombs (symbol MFRC), together with their corresponding statistical error bars.

The orientation of the individual MFR normals obtained in the analysis are certainly corrupted by the MP non-planarity. However, for an ideal 2-D discontinuity, the normals provided by the planar MFR technique would be contained in one plane, the same that contains also the individual MVAB normals. It is remarkable that in our case, in spite of the mentioned problems with the data, the constrained MFR normals lie very close, basically within the statistical errors, to the plane of the normals identified in Sect. 2.1 (see parameter $\Omega$ in the Table 1). In fact, if we proceed as described at the end of Sect. 2.1 and compute the invariant direction based entirely on the individual MFR normals, we obtain a direction only $3^{\circ}$ away off the invariant direction computed there. Thus, the MFR results are consistent with the earlier conclusion of a 2-D, non-planar MP.

\subsection{Extracting the timing information}

For extracting the timing information, a procedure like that described in Haaland et al. (2004), with some alterations, has been followed. Firstly, the magnetic field data recorded by 


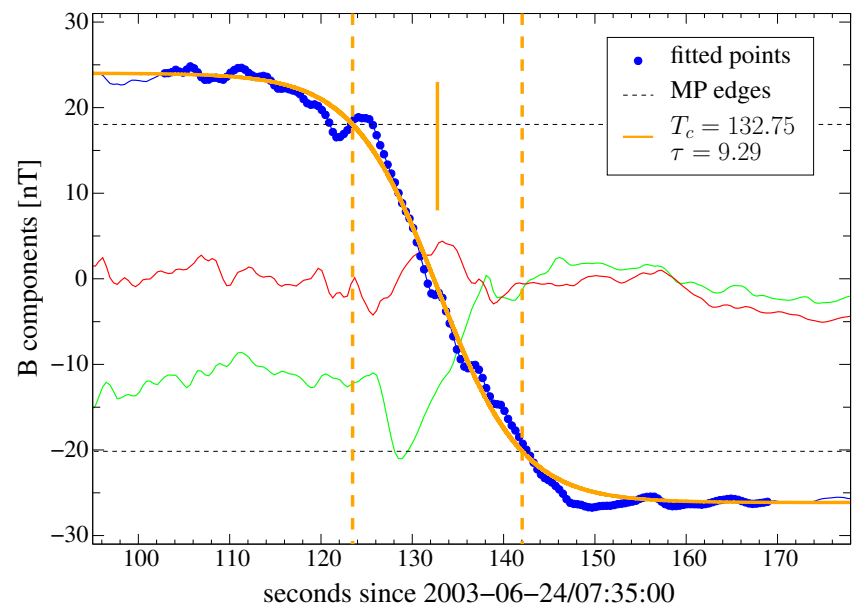

Fig. 5. C1 magnetic field components along the directions of minimum magnetic field variance (red trace), of maximum magnetic field variance (blue trace) and along $\langle\boldsymbol{B}\rangle$ (green trace). The timing information, shown in the legend box, is obtained by fitting the points (in blue) corresponding to the maximum variance component with a hyperbolic tangent function, shown in orange. More details are provided in the text.

each satellite was transformed into the local MP frame provided by the constrained MVAB analysis, having the axes along the directions providing the maximum magnetic variance, the minimum magnetic variance and along the direction of $\langle\boldsymbol{B}\rangle$. Then, the maximum magnetic variance component was fitted with a hyperbolic tangent function

$B(t)=B_{m}-\frac{1}{2} \Delta B \tanh \left[\frac{t-T_{c}}{\tau}\right]$

in order to determine the crossing time that characterizes the MP transition in the global sense. Here $B_{m}$ is the zero level of the tanh function, $\Delta B$ is the total magnetic jump between the magnetosheath and magnetopause asymptotic levels, $T_{c}$ represents the central time and $\tau$ designates the profile halfwidth. Note that, because of a non-planar MP, we expect that the direction providing the minimum magnetic field variance will be different from one satellite to another and therefore each fit will be performed in a slightly different reference frame.

Next, a definition for the MP extent as the central region where the fit changes from $B_{m}-\frac{1}{2} \Delta B \tanh (-1)$ to $B_{m}-\frac{1}{2} \Delta B \tanh (1)$ magnetic levels was adopted. This corresponds to a fraction of $\sim 0.762$ of the total magnetic jump $\Delta B$. According to this definition, the satellite encounters the MP leading edge at $T_{c}-\tau$ and the MP trailing edge at $T_{c}+\tau$. These two instants of time will be used directly in the method (not the central crossing time $T_{c}$, as in Haaland et al. 2004).

As an illustration of the procedure described above, Fig. 5 presents the $\mathrm{C} 1$ magnetic field components in the reference frame related to the corresponding MVAB normal. The magnetic field components along the direction providing the
Table 2. Satellite relative positions and timing for the analyzed event. For each satellite the components along the $\boldsymbol{l},\langle\boldsymbol{n}\rangle_{\mathrm{MVAB}}$, and $\boldsymbol{t}$ directions (see the text) are provided, together with the MP central crossing time $T_{c}$, and crossing duration $\tau$. The $\mathrm{C} 2$ position is taken as origin of the reference frame and the $T_{c}$ quantities are computed with respect to the $\mathrm{C} 2$ central crossing time.

\begin{tabular}{crrrrrrr}
\hline & \multicolumn{3}{c}{ position $[\mathrm{km}]$} & & \multicolumn{2}{c}{ timing $[s]$} \\
\cline { 2 - 3 } \cline { 7 - 8 } & \multicolumn{1}{c}{$R_{l}$} & \multicolumn{1}{c}{$R_{n}$} & \multicolumn{1}{c}{$R_{t}$} & & $T_{c}$ & $\tau$ \\
\hline $\mathrm{C} 2$ & 0.0 & 0.0 & 0.0 & & 0.0 & 6.44 \\
$\mathrm{C} 4$ & -90.6 & 170.9 & 167.6 & & 2.75 & 7.27 \\
$\mathrm{C} 3$ & -1664.9 & 541.9 & 7306.5 & & 34.87 & 8.91 \\
$\mathrm{C} 1$ & -1954.5 & 588.1 & 7506.0 & & 37.81 & 9.29 \\
\hline
\end{tabular}

maximum magnetic field variance, the minimum magnetic field variance and along the direction of $\langle\boldsymbol{B}\rangle$ are shown by blue, red and green lines respectively. The points included in the fit are shown in blue. The parameters $T_{c}$ (indicated by the vertical yellow segment) and $\tau$ (the half-distance between the vertical yellow dashed lines) determined in the analysis are provided in the insert. The horizontal dashed lines represent the magnetic field levels associated with the MP edges.

Table 2 presents the satellites' relative positions and the timing information for the 24 June 2004 event. The quantities $R_{l}, R_{n}, R_{t}$, are the separation distances along the invariant direction $\boldsymbol{l}$, along $\langle\boldsymbol{n}\rangle_{\mathrm{MVAB}}$, and along $\boldsymbol{t}=\langle\boldsymbol{n}\rangle_{\mathrm{MVAB}} \times \boldsymbol{l}$, respectively. We choose as reference the position of $\mathrm{C} 2$, i.e. the first satellite detecting the MP. The central crossing times $T_{c}$, computed with respect to the time when $\mathrm{C} 2$ detects the MP center, and the crossing durations $\tau$ are shown. From Table 2 one can observe that the satellites MP transitions are well separated, the minimum separation being $2.75 \mathrm{~s}$.

\subsection{The results from planar timing analysis}

When carrying out the CVA and CTA timing analysis (Haaland et al., 2004), in order to stay fully consistent with the planar assumption, where a single normal direction characterizes the event, the timing information was extracted from fits similar to that presented in Fig. 5 but on magnetic traces in a common reference frame, related to the $\langle\boldsymbol{n}\rangle_{\text {MVAB }}$ direction $^{2}$.

In Fig. 4 the CVA and CTA normals are indicated with red and golden open circles respectively. Their orientation is well apart from the $\langle\boldsymbol{n}\rangle_{\mathrm{MVAB}}$ direction, i.e. $\sim 32^{\circ}$ and $\sim 38^{\circ}$ for the CTA and the CVA normals, respectively. Considering the regular magnetic profiles seen by all satellites and the good quality of the fits, resulting in a trustworthy timing

\footnotetext{
${ }^{2}$ In our particular case this aspect has practically no influence: we can equally well use the timing information from the preceding section and obtain the same conclusions.
} 
information, this discrepancy is very significant, suggesting again a non-planar geometry for the MP.

To save the planar assumption, one could argue that, perhaps, neither the constant thickness nor the constant velocity assumptions are valid for this event but, instead, there is a variable normal velocity and the MP has different thickness at different points on its surface. We investigated this possibility by imposing different orientations for a planar layer, around the direction of $\langle\boldsymbol{n}\rangle_{\mathrm{MVAB}}$. Then, the central crossing times and the relative positions of the satellites along the imposed normal allow us to determine the MP normal velocity evolution ${ }^{3}$. By proceeding in this way, and imposing for example $\langle\boldsymbol{n}\rangle_{\text {MVAB }}$ as normal direction, one obtains unreasonably large variations in the MP thickness from one satellite to another. For example, between $\mathrm{C} 3$ and $\mathrm{C} 1$, separated by only $202 \mathrm{~km}$ in the MP plane, a MP thickness variation of $183 \mathrm{~km}$, from $254 \mathrm{~km}$ to $437 \mathrm{~km}$, was obtained. A similar behavior was noticed for other directions close to the $\langle\boldsymbol{n}\rangle_{\text {MVAB }}$.

An interesting observation related to Fig. 4 is the following: when the MVAB technique, which assumes a planar geometry, is applied on a 2-D discontinuity, the normal orientation will be affected by the non-planarity. However, the information about the 2-D symmetry contained in the actual magnetic measurements (ideally, the measurements will not vary along the invariant direction) is reflected in the result, and the normal will lie in the plane perpendicular to the invariant direction. The same consideration applies to the planar MFR technique as well. By contrast, because the techniques employed in this subsection rely solely on the satellites position and on the timing information, a 2-D MP investigated with the standard CVA or CTA will not produce, in general, normals in the plane perpendicular to $\boldsymbol{l}$.

\section{Presentation of the new method}

The new method assumes that the MP can be locally modelled as a layer of constant thickness that has either a parabolic or a cylindrical shape, that does not change along the direction perpendicular to the plane of the normals (the invariant direction). In the plane of the normals we allow for the layer to have either a unidirectional movement or independent motions along two mutually perpendicular directions. Any motion along the third, invariant direction has no meaning because it causes no change. Note that this model can describe two main features of the non-planar MP: its inward-outward motion and, by allowing for a second degree of freedom, the large-scale waves that often travel along its surface.

There are actually two techniques that we developed for determining the crossing parameters of a 2-D, non-planar discontinuity. The first is based, apart from the knowledge of invariant direction, solely on the timing information, thus

\footnotetext{
${ }^{3} \mathrm{~A}$ second degree polynomial has been used to describe the MP normal velocity time-dependence.
}

being a generalization of the planar CTA technique. The second technique uses simultaneously the timing information and the $\nabla \cdot \boldsymbol{B}=0$ conservation law, providing solutions that comply with the requirement of minimum normal magnetic field variance. The two techniques, which we will refer to as the straight timing analysis and the combined timing $-M V A B$ analysis, are briefly described in Sect. 3.1 and Sect. 3.2, respectively. Additional details are given in Appendix A.

\subsection{Straight timing analysis}

Let us suppose that the necessary input parameters, i.e. the invariant direction and the timing information, were extracted from the data, as shown in Sect. 2.3 for the 24 June 2003 event. Then, mathematically, in each implementation of the model (parabolic or cylindrical geometry, one or two degrees of freedom for the MP movement), a system of eight equations can be established, i.e. two equations for each satellite. This corresponds to the conditions that, during its motion, the leading and trailing edges of a 2-D MP oriented along the invariant direction encounters the satellites' positions according to the timing information. Note that the method uses the times when the satellites detect the MP leading and trailing edges, and not the time when the MP center is detected, contrary to the planar CTA and CVA methods.

Figures 6 and 7 schematically show cross-sections of a parabolic, respectively cylindrical, MP at successive times (more explanations related to the figures are given in Appendix A). The invariant direction $l$ points into the page. The unknowns to be found from the above mentioned system of eight equations are:

- the spatial scale of the structure, given by the quadratic coefficient of the parabola describing the MP profile, in the parabolic case, or by the MP inner radius of curvature, in the cylindrical case;

- the MP thickness;

- the angle in the plane of the normals specifying the direction of primary MP motion (see below);

- the initial position of the structure, given by two coordinates in the plane of the normals, specifying the MP leading edge apex position, in the parabolic case, or the MP center of curvature, in the cylindrical case;

- three coefficients describing the velocity-time dependence, assumed to be polynomial.

When the model allows two degrees of freedom for the MP movement, the last three parameters describe a uniformly accelerated displacement along one direction, called hereafter the primary direction, and a constant velocity motion along the perpendicular direction, called hereafter the secondary direction. When the MP is allowed to move only along one direction (called primary as well), the last three parameters 


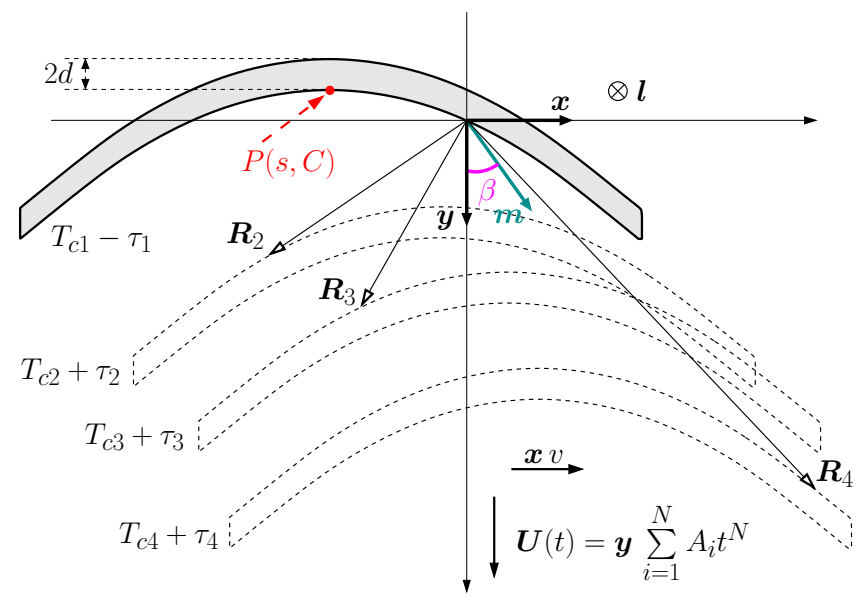

Fig. 6. MP as a parabolic layer, in cross-sections at successive moments. The unit vector $\boldsymbol{l}$, indicating the invariant direction, points into the paper. The Cluster satellites are located at $\boldsymbol{R}_{i}, i=1 \ldots 4$, with $\boldsymbol{R}_{1} \equiv 0$. At the initial time, $T_{c 1}-\tau_{1}$, the parabola describing the MP leading edge has the apex at $P(s, C)$, and encounters the first satellite at the origin; at the other three times shown, at $T_{c i}+\tau_{i}, i=2 \ldots 4$, the MP trailing edge is detected by the second, third and fourth satellite, respectively. According to the model, the MP primary movement, with a polynomial time dependence for velocity, takes place along $\boldsymbol{y}$, parallel to the parabola's axis. The secondary movement, allowed in one variant of the model, occurs with constant velocity in the perpendicular direction. Details about the quantities shown in this figure are discussed in Appendix A.

characterize the structure's velocity, acceleration and change in the acceleration, respectively.

\subsection{Combined timing - MVAB analysis}

The straight timing analysis presented in the previous subsection can be improved if one takes advantage of the fact that the primary direction of MP movement is fully described by one parameter, i.e. an angle in the plane of the normals.

Based on that, one can impose different values for this parameter, and solve the system of eight equations from the previous subsection with this condition. For each solution that is found, corresponding to one angle, the magnetic field variance along the instantaneous, i.e. geometrical, MP normal at each satellite can be computed.

$\sigma_{i}^{2}=\frac{1}{M_{i}} \sum_{m=1}^{M_{i}}\left|\left(\boldsymbol{B}_{i}^{(m)}-\langle\boldsymbol{B}\rangle_{i}\right) \cdot \boldsymbol{n}_{2 \mathrm{D}, \mathrm{i}}^{(m)}\right|^{2} \quad i=1 \ldots 4$

Here $\boldsymbol{B}_{i}^{(m)}, m=1 \ldots M_{i}$, represent the magnetic measurements taken by satellite $i$, and $\boldsymbol{n}_{2 \mathrm{D}, \mathrm{i}}^{(m)}$ designates the 2-D, instantaneous geometrical normal at the same satellite. The superscript $m$ indicates that these normals are changing in time and therefore their orientation at the time when the $\boldsymbol{B}^{(m)}$ measurement is taken will be considered in Eq. (1).

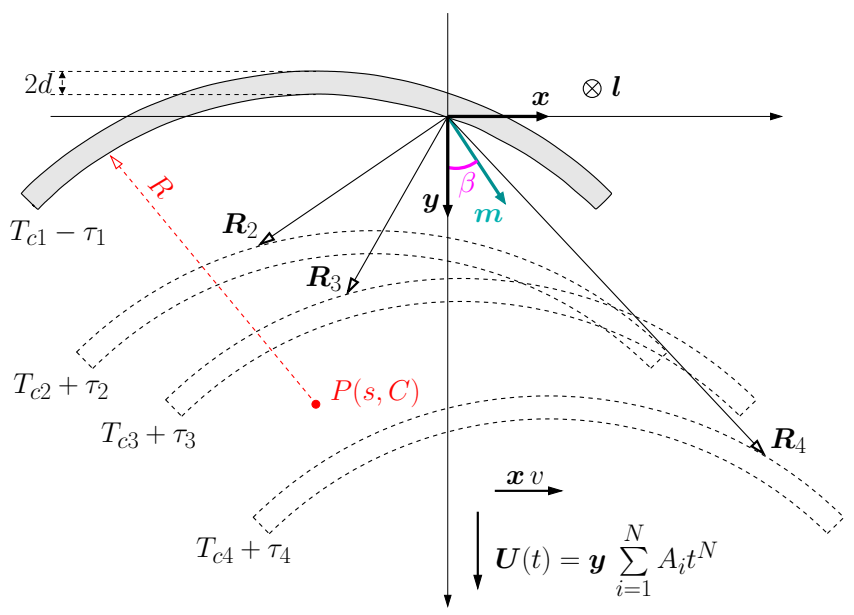

Fig. 7. Figure similar to Fig. 6 but corresponding to a cylindrical MP layer. Now the coordinates $(s, C)$ indicate the MP center of curvature $P$ at the initial time $T_{c 1}-\tau_{1}$. Details about the quantities shown in this figure are discussed in Appendix A.

If the angle defining the direction of MP movement is increased stepwise, within the range $[-\pi, \pi]$, one can select that direction of MP movement for which the global normal magnetic field variance is minimum. Here by "global", a weighted average over the four satellites is meant, i.e. $\sigma_{\text {global }}^{2}=\sum_{i=1}^{4} p_{i} \sigma_{i}^{2}$. Details about how to define the corresponding weighting factors $p_{i}$ are provided in Appendix A (see Eq. A5), the underlying argument being the following: if the magnetic field measured at one satellite is, by whatever reason, more perturbed than at the other, i.e. if it implies higher magnetic field fluctuations, then its contribution to the global magnetic field variance is reduced by the weighting factor.

The solution obtained in this way has two advantages: first, it combines in a self-consistent way the timing information with the requirements of $\nabla \cdot \boldsymbol{B}=0$. Second, because the angle specifying the direction of MP movement is now set from outside, four coefficients, instead of three, are available to describe the MP velocity-time dependence, providing an improved description of the MP motion.

\section{Solutions provided by the 2-D, non-planar method}

When applying the various implementations of the 2-D, nonplanar MP method introduced in Sect. 3 to the 24 June 2003 event, a total of six valid solutions have been obtained. In what follows a certain nomenclature to designate a particular solution will be used. Each name will indicate the model adopted for the MP ("P" for parabolic and "C" for cylindrical), the number of degrees of freedom allowed in the MP movement ("1" or "2") and the implementation of the method ('TA' for the straight timing analysis and "OpTA" for the optimized method, combining timing analysis and 


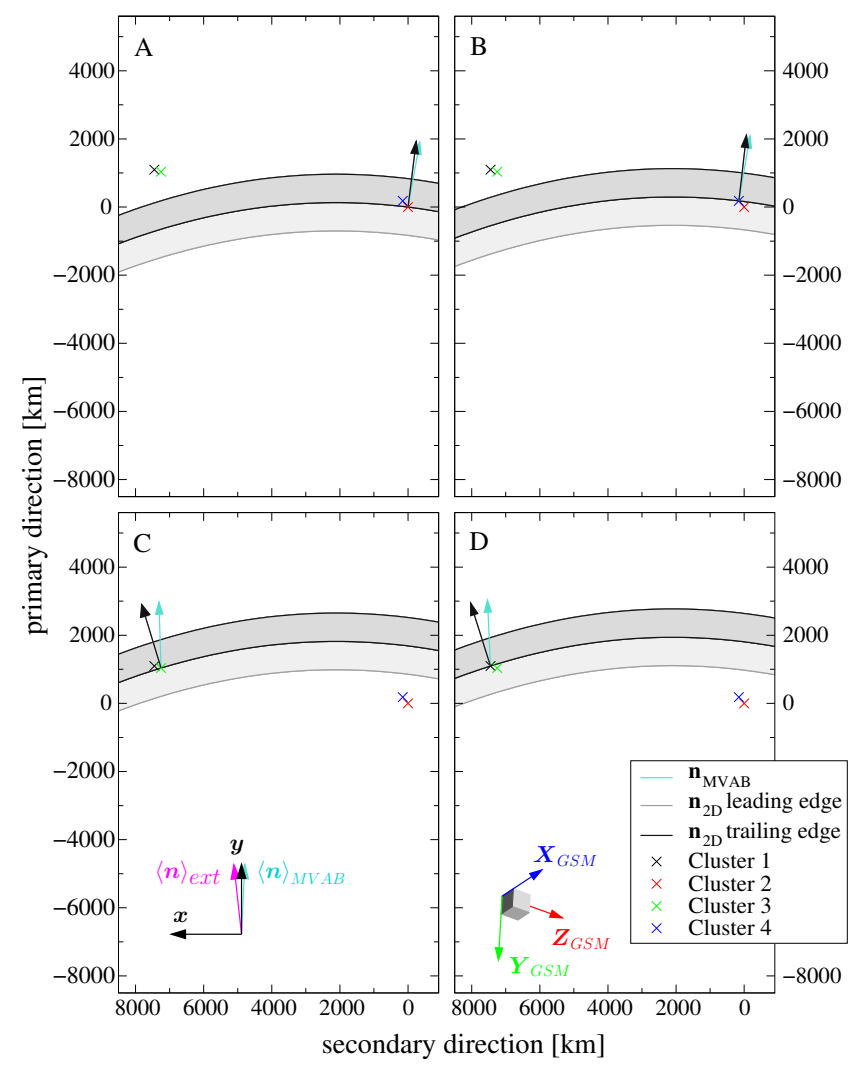

Fig. 8. The 2-D, non-planar MP motion in the plane perpendicular to the invariant direction, according to the P1_OpTA solution. For a description of this picture see the text.

MVAB technique). For the cylindrical MP having 2 degrees of freedom, two solutions have been found with the combined timing-MVAB technique; they will be differentiated by the suffix "A" and "B", respectively.

This section presents three exemplary 2-D, non-planar solutions, one based on the parabolic model, i.e. P1_OpTA shown in Fig. 8, and two on the cylindrical model, i.e. C2_OpTA_A and C2_OpTA_B shown in Fig. 9 and Fig. 10, respectively. For a detailed presentation of all 2-D solutions, the reader is referred to Blagau (2007).

In Figs. 8, 9, and 10, the spacecraft positions are indicated by crosses, colored according to the mission convention. The panels A, B, C, and D are associated with the satellite $\mathrm{C} 2, \mathrm{C} 4$, $\mathrm{C} 3$, and $\mathrm{C} 1$ respectively, and present the MP cross-sections in the plane perpendicular to the invariant direction $\boldsymbol{l}$ at two moments: when the corresponding satellite enters the layer (i.e. at $T_{c i}-\tau_{i}, i=1 \ldots 4$, in light gray) and when it leaves the layer (i.e. at $T_{c i}+\tau_{i}$, in darker gray). The MP external geometrical normals ${ }^{4}, \boldsymbol{n}_{2 \mathrm{D}}$, at the satellite position for these

\footnotetext{
${ }^{4}$ Here by "external geometrical" we mean pointing into the opposite direction of the center of curvature, not the direction outward from the magnetosphere. Whether the two directions are parallel or anti-parallel depends on the center of curvature location, i.e. ei-
}

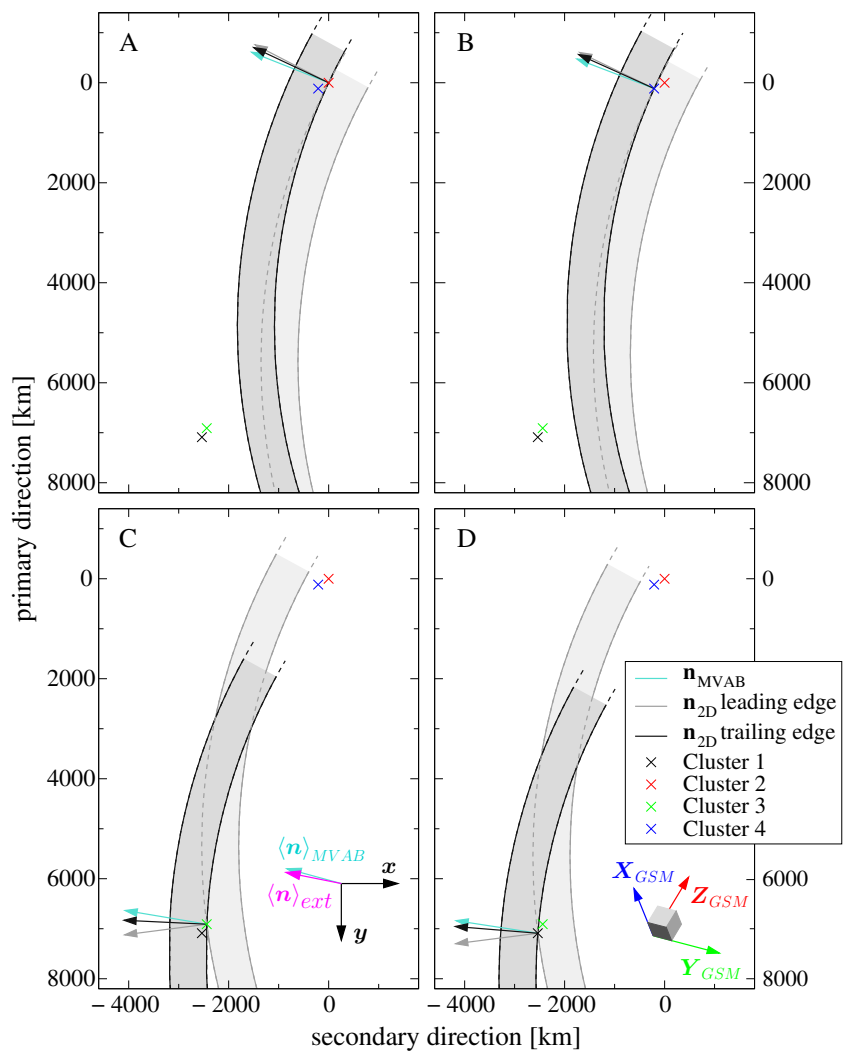

Fig. 9. The 2-D, non-planar MP motion in the plane perpendicular to the invariant direction, according to the C2_OpTA_A solution. For a description of this picture see the text.

two moments are shown, together with the individual normal obtained from the planar, nested MVAB technique (in cyan; actually the projection of this normal on the plane perpendicular to the invariant direction is shown).

The primary movement takes place along $\boldsymbol{y}$, i.e. along the vertical. In the last two solutions, see Figs. 9 and 10, the MP has also a secondary motion, with constant velocity, along $\boldsymbol{x}$. In panel (c), besides the $\boldsymbol{y}$ and $\boldsymbol{x}$ axes, two other directions are shown. In light blue is the average of the planar, individual normals obtained in the nested MVAB analysis, $\langle\boldsymbol{n}\rangle_{\mathrm{MVAB}}$, chosen also as reference direction in Figs. 3 and 4 from Sect. 2. For the second direction, one first computes for each satellite the individual $\boldsymbol{n}_{2 \mathrm{D}}^{\text {ave }}$ normal, i.e. the average (over the crossing duration) external geometrical normal based on the 2-D solution. The unit vector $\langle\boldsymbol{n}\rangle_{\mathrm{ext}}$, shown in magenta, is along the vectorial mean of the four $\boldsymbol{n}_{2 \mathrm{D}}^{\text {ave normals. In }}$ panel (d), the orientation of $\boldsymbol{X}_{\mathrm{GSM}}, \boldsymbol{Y}_{\mathrm{GSM}}$ and $\boldsymbol{Z}_{\mathrm{GSM}}$, i.e. the unit vectors defining the GSM reference frame, as seen by looking against the invariant direction $\boldsymbol{l}$ (pointing into the page) are indicated.

ther inside or outside the magnetosphere, which can be established based on the character of the crossing (inbound or outbound). 


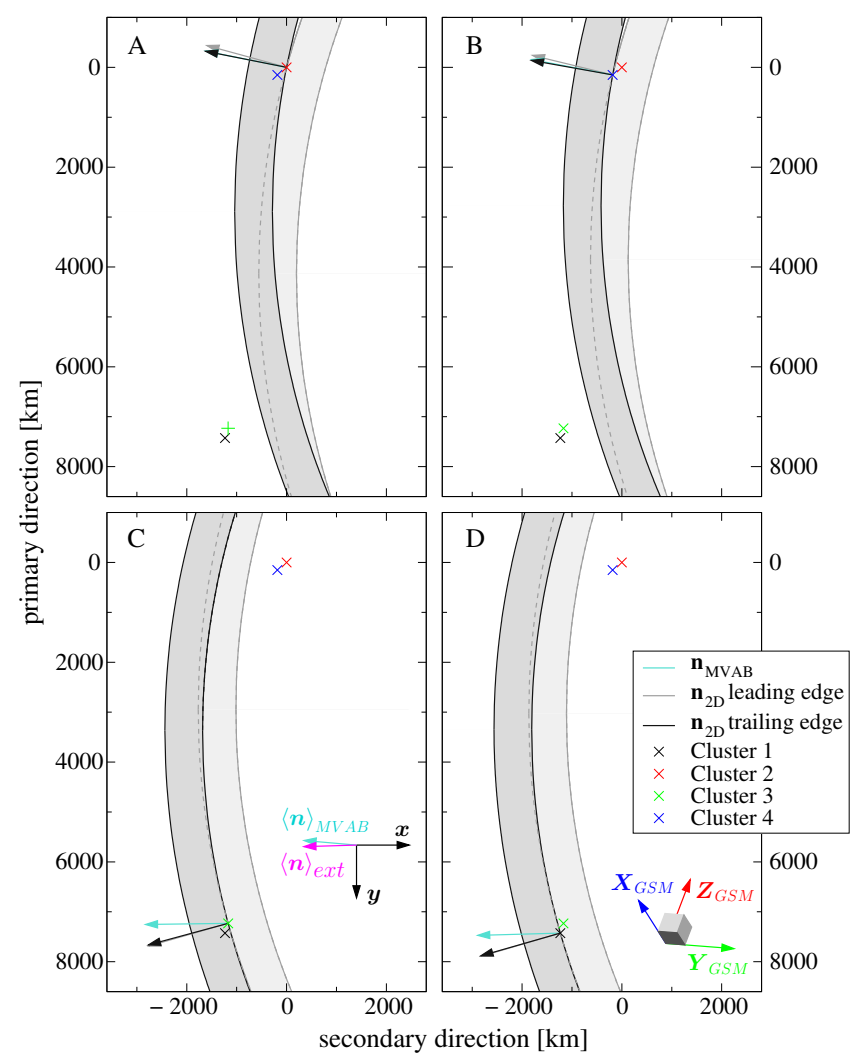

Fig. 10. The 2-D, non-planar MP motion in the plane perpendicular to the invariant direction, according to the C2_OpTA_B solution. For a description of this picture see the text.

Since, by definition, $\langle\boldsymbol{n}\rangle_{\text {ext }}$ is oriented into the opposite direction of the center of curvature, the in-bound character of the transition (see the beginning of Sect. 2) implies a convex shape for the MP depicted in Figs. 8, 9 and 10 (the same result has actually been obtained from all acceptable 2-D solutions corresponding to the 24 June 2003 event; see Blagau, 2007). The solution P1_OpTA from Fig. 8 is consistent with a local, stationary bulge on the MP surface, modelled by a parabolic layer moving (outward) exclusively along the $\boldsymbol{y}$ direction. The other two cases, shown here in Fig. 9 and Fig. 10, indicate a traveling wave on the MP surface: the general outward motion occurs with constant velocity along the $\boldsymbol{x}$ direction, while a more complicated, back and forth movement takes place along the $\boldsymbol{y}$ direction. ${ }^{5}$

The solution C2_OpTA_A from Fig. 9 implies a reencounter of the MP by the first pair of satellites (C2 and C4) at the end of the event, i.e. around the time when the second

\footnotetext{
${ }^{5}$ Note that for these two solutions, the primary direction $y$ is not oriented close to the MP normal but it is roughly perpendicular to it, i.e. opposite of the situation depicted in Fig. 7. This is because we did not impose any restriction of this kind in the model; the orientation of $\boldsymbol{x}$ and $\boldsymbol{y}$ axes is obtained exclusively by solving the conditions of MP encounter.
}

pair of satellites ( $\mathrm{C} 3$ and $\mathrm{C} 1$ ) finish their transitions. The return is not seen in the data and strictly speaking means a disagreement with the physical situation. This aspect could be reconciled if one limits in an appropriate way the solution's range of validity, like has been done in Fig. 9, where the upper part of the MP was not sketched. No such limitation was needed for the P1_OpTA and C2_OpTA_B solutions.

It is worth mentioning that the C2_OpTA_A solution, shown in Fig. 9, and the C2_TA solution, not shown here, are practically identical. This is remarkable, meaning that the method based solely on the timing information already provides a result optimized with respect to the $\nabla \cdot \boldsymbol{B}=0$ conservation law.

The parameters that characterize all the valid 2-D solutions corresponding to 24 June 2003 event will be presented in Sect. 6, when also the normal magnetic field variance and the average normal magnetic field quantities implied by each solution are shown. It will be clear that, in spite of the apparent diversity, the MP transition parameters (orientation, normal velocity, thickness and curvature) obtained by the various implementations are actually not very different.

\section{Detailed presentation of one 2-D solution}

In this section, the solution C2_OpTA_A from Fig. 9 (i.e. the first solution found with the combined timing-MVAB technique for a cylindrical MP having two degrees of freedom) will be discussed in detail and compared with the results provided by the planar methods like MVAB, MFR, timing analysis, and HT analysis. Table 3 summarizes this comparison both in terms of individual quantities, i.e. corresponding to each satellite, as well as in terms of the average, or "global" ones. Below a brief description of the parameters shown in this table is given.

The Planar methods part of Table 3 presents first the results of the constrained MVAB analysis on eleven nested intervals (see Sect. 2.1) in terms of normal magnetic field variance and average normal magnetic field component $\left\langle B_{n}\right\rangle$. The next two lines show similar quantities obtained by applying a constrained MVAB analysis on the central intervals $\left[T_{c i}-\tau_{i}, T_{c i}+\tau_{i}\right]$. Here the constraint $\boldsymbol{l} \cdot \boldsymbol{n}=0$ has been used, thus eliminating in the analysis the influence of magnetic fluctuations perpendicular to the plane of the normals, in accordance with the 2-D geometry assumption for the MP. Further on, the normal MP velocity is presented, as determined by combining the nested MVAB normals with the HT analysis (see Sect. 5.3), by applying the MFR technique (see Sect. 2.2), and by applying the two variants of the planar timing analysis, i.e. CTA and CVA (see Sect. 2.4).

The last line of the Planar methods part presents the MP normal velocity at each pair of consecutive satellites $i, i+1$, with $i=1 \ldots 3$, when the boundary is assumed to have locally, i.e. between the satellites crossing times $T_{c, i}$ and $T_{c, i+1}$, a stable, planar geometry. For the local MP normal we took 
Table 3. Comparison between the C2_OpTA_A solution and the planar results provided by MVAB, HT, MFR, and timing analysis techniques. Quantities corresponding to each satellite as well as average or global ones are shown. With smaller blue numbers, the global values of normal magnetic field variance and of $\left\langle B_{n}\right\rangle$ based only on data from $\mathrm{C} 1, \mathrm{C} 3$ and $\mathrm{C} 4$ are indicated. More explanations are provided in the text.

\begin{tabular}{|c|c|c|c|c|c|}
\hline & average & $\mathrm{C} 2$ & $\mathrm{C} 4$ & $\mathrm{C} 3$ & $\mathrm{C} 1$ \\
\hline \multicolumn{6}{|l|}{ Planar methods } \\
\hline \multicolumn{6}{|l|}{ MVAB on nested intervals } \\
\hline Magnetic variance $[\mathrm{nT}]^{2}$ & 3.593 .45 & 4.09 & 4.64 & 2.58 & 2.89 \\
\hline$\left\langle\mathrm{B}_{\mathrm{n}}\right\rangle[\mathrm{nT}]$ & $0.0 \quad 0.0$ & 0.0 & 0.0 & 0.0 & 0.0 \\
\hline \multicolumn{6}{|l|}{ MVAB on central intervals } \\
\hline Magnetic variance $[n T]^{2}$ & 2.722 .70 & 2.82 & 1.16 & 2.79 & 4.21 \\
\hline$\left\langle\mathrm{B}_{\mathrm{n}}\right\rangle[n T]$ & $-1.7-1.6$ & 1.4 & -1.8 & -1.4 & -1.6 \\
\hline \multicolumn{6}{|l|}{ MVAB and $\mathrm{HT}$ analysis } \\
\hline $\boldsymbol{V}_{\mathrm{HT}} \cdot \boldsymbol{n}_{\mathrm{MVAB}}^{\text {nested }}[\mathrm{km} / \mathrm{s}]$ & 22.3 & 86.6 & -5.8 & -14.4 & 22.6 \\
\hline \multicolumn{6}{|l|}{ MFR analysis } \\
\hline $\mathrm{V}_{\mathrm{MFR}}[\mathrm{km} / \mathrm{s}]$ & 136 & 139 & 182 & 56 & 167 \\
\hline \multicolumn{6}{|l|}{ Timing analysis } \\
\hline $\mathrm{V}_{\mathrm{CTA}}[\mathrm{km} / \mathrm{s}]$ & 78.7 & 94.4 & 81.4 & 70.5 & 68.6 \\
\hline $\mathrm{V}_{\mathrm{CVA}}[\mathrm{km} / \mathrm{s}]$ & 87.1 & 87.1 & 87.1 & 87.1 & 87.1 \\
\hline \multicolumn{6}{|c|}{ Timing analysis on spacecraft pairs } \\
\hline $\mathrm{V}_{\text {pair }}[\mathrm{km} / \mathrm{s}]$ & 15.5 & \multicolumn{2}{|c|}{55.3} & \multicolumn{2}{|c|}{$.4 \quad 22.9$} \\
\hline \multicolumn{6}{|l|}{ C2_OpTA_A } \\
\hline \multicolumn{6}{|l|}{ Normals orientation } \\
\hline$\varangle\left(\boldsymbol{n}_{2 \mathrm{D}}^{\mathrm{ave}}, \boldsymbol{n}_{\mathrm{MVAB}}^{\text {nested }}\right)_{\perp \boldsymbol{l}}$ & 7.9 & 4.4 & 3.7 & 12.6 & 10.9 \\
\hline$\Delta \boldsymbol{n}_{2 \mathrm{D}} \quad[\mathrm{deg}]$ & 6.4 & 2.1 & 1.6 & 10.1 & 11.9 \\
\hline \multicolumn{6}{|l|}{ Normal velocity } \\
\hline $\boldsymbol{v}_{2 \mathrm{D}}^{\text {ave }}[\mathrm{km} / \mathrm{s}]$ & 47.5 & 57.4 & 50.9 & 41.5 & 39.9 \\
\hline \multicolumn{6}{|c|}{ Field quantities using $\boldsymbol{n}_{2 \mathrm{D}}$ and $\boldsymbol{v}_{2 \mathrm{D}}$} \\
\hline Magnetic variance $[\mathrm{nT}]^{2}$ & 2.502 .26 & 3.32 & 1.39 & 2.28 & 3.14 \\
\hline$\left\langle B_{n}\right\rangle \quad[\mathrm{nT}]$ & $-0.7-1.1$ & 0.6 & -1.2 & -1.1 & -1.0 \\
\hline Magnetic variance weighting fa & actors & 0.22 & 0.28 & 0.22 & 0.28 \\
\hline
\end{tabular}

the average of the coresponding MVAB normals and therefore the $\mathrm{V}_{\text {pair }}$ quantities were computed based on the satellites separation along that direction and on the difference $T_{c, i+1}-T_{c, i}$. The MP global velocity, presented in the first column, is a weighted average which takes into account that each $V_{\text {pair }}$ is valid for a different time length.

In the C2_OpTA_A part of Table 3, all quantities refer to the central intervals. The first line presents the angle between the $\boldsymbol{n}_{2 \mathrm{D}}^{\text {ave }}$ and the individual MVAB normals, projected on the plane perpendicular to the invariant direction $\boldsymbol{l}$, and reflects the difference in normal prediction by the two methods. The second line shows the change in orientation of the instantaneous geometrical normals, $\boldsymbol{n}_{2 \mathrm{D}}$, during the crossing interval, measuring how the MP movement and curvature influence the normal detected by the satellites. The average (over the crossing duration) MP normal velocity, $\boldsymbol{v}_{2 \mathrm{D}}^{\text {ave }}$, is shown on the following line. Next, the normal magnetic field variance and the values for $\left\langle B_{n}\right\rangle$ are presented.

The weighting factors, used to compute the global value of the normal magnetic field variance, are shown on the last line. They were derived based on the arguments presented in Appendix A (see the discussions related to Eq. A5).

\subsection{The investigation of normal magnetic field variance}

When judging whether the planar or the 2-D method provides solutions that comply better with the requirement of minimum normal magnetic field variance, one has to take into account that each technique uses different data intervals: the planar MVAB is based on data intervals large enough to include the whole magnetic field rotation seen at the MP, whereas in the 2-D method only the central intervals, $\left[T_{c i}-\tau_{i}, T_{c i}+\tau_{i}\right]$, are used, in accordance with the system of conditions describing the satellites encounter with the MP edges. Therefore, it is instructive to investigate how the solutions provided by the two techniques behave starting from the central intervals up to the largest intervals used in the nested MVAB analysis (see Sect. 2.1).

Such an investigation has been carried out in Appendix B. There, using intervals centred on $T_{c i}$ that progressively increase in size, we analyzed the evolution of the normal magnetic field variance and of the average normal magnetic field component $\left\langle B_{n}\right\rangle$, based on three sets of normals: the individual, planar normals obtained from the nested MVAB analysis, the 2-D, instantaneous, geometrical normals corresponding to the C2_OpTA_A solution and the normals provided by a constrained, to $\boldsymbol{l} \cdot \boldsymbol{n}=0$, MVAB analysis performed for that particular interval. Note that the latter normals, called here and in Appendix B the planar, interval-specific MVAB normals, are changing with the interval of analysis according to the MVAB theory.

The analysis shows that, even for intervals well beyond the validity range of the $2-\mathrm{D}$ solution, i.e. $\left[T_{c i}-\tau_{i}, T_{c i}+\tau_{i}\right]$, the global magnetic field variance based on the 2-D, geometrical normals is smaller than the same quantity based on the planar, interval-specific MVAB normals (which is, evidently, smaller than that based on the normals provided by the nested MVAB analysis). Therefore one can definitely say that, for the above mentioned intervals, the 2-D solution C2_OpTA_A performs better than the planar MVAB solution from normal magnetic field variance point of view.

However, we think that when judging one particular solution provided by the 2-D, non-planar method it is not necessary to adopt such a strict criterion, namely that the global 
magnetic field variance based on the 2-D, geometrical normals to be smaller than that based on the planar, intervalspecific MVAB normals. The latter normals are not correlated between the satellites and, for an individual satellite, change their direction from one interval to the other independent of the MP dynamics. By contrast, the 2-D, geometrical normals are linked at each moment by the geometry proposed for the MP (parabolic or cylindrical) and evolve in time according to the global MP dynamics. Therefore, it seems reasonable to consider that a 2 -D solution providing values for the global magnetic field variance greater than (but close to) that based on planar, interval-specific MVAB normals is still performing very well from the magnetic field variance perspective, and that overall, it is better than the planar solution.

If we take the central intervals and compute the global magnetic field variance based on the normals provided by the planar, nested MVAB analysis, a value of $4.12[\mathrm{nT}]^{2}$ is obtained. This would roughly correspond to the situation described in Sect. 2.1, of a planar MP that changes its orientation between the crossing of the first pair of satelites and that of the second pair of satellites. Because of the significantly higher value it implies, higher than for any solution obtained with the 2-D method (see Table 5 from Sect. 6), one can clearly see that such an alternative interpretation of the data has to be rejected. ${ }^{6}$

\subsection{Orientation of the 2-D, non-planar magnetopause}

The second part of Appendix B investigates how the relative orientation between the three sets of normals mentioned in Sect. 5.1 (i.e. the normals provided by the planar, nested MVAB, the 2-D geometrical normals, and the planar, interval-specific MVAB normals) changes, when progressively larger intervals centred on $T_{c i}$ are used.

Based on this analysis we concluded that in case of $\mathrm{C} 2$ the MVAB results corresponding to the central intervals, $\left[T_{c i}-\tau_{i}, T_{c i}+\tau_{i}\right]$, are significantly affected by local, internal irregularities (magnetic islands, noise etc.). This is the reason why, in Table 3 and in Table 5 from Sect. 6, the global normal magnetic field variance based both on data from all four satellites (black numbers) as well as only on data from $\mathrm{C} 1, \mathrm{C} 3$ and $\mathrm{C} 4$ (smaller blue numbers) is shown.

In Table 3, the first line from the C2_OpTA_A part shows how different the $\boldsymbol{n}_{2 \mathrm{D}}^{\text {ave }}$ normals are with respect to the individual MVAB normals obtained in the nested analysis. Based on these values and on the similar ones provided by the other 2-D MP solutions ${ }^{7}$, one would be inclined to say that all non-

\footnotetext{
${ }^{6}$ In fact, in the combined timing-MVAB analysis, for certain directions of MP movement solutions were found that imply a very large radius of curvature for the MP and global magnetic field variance around this value, but they were rejected by the optimization criterion employed in this technique.

${ }^{7}$ For example, the angles between the two normals corresponding to P1_OpTA are $[3.2,3.1,15.1,14.9]$, and to C2_OpTA_B they are $[1.5,0.7,14.6,14.4]$ at the $\mathrm{C} 2, \mathrm{C} 4, \mathrm{C} 3$ and $\mathrm{C} 1$ satellite, respec-
}

Table 4. Results from the HT analysis. For each satellite one shows the interval of analysis (in seconds from 07:36:00 UT), the correlation coefficient and the regression line slope between the electric field in the starting reference frame $\boldsymbol{E}_{i}$ and the convection electric field $\boldsymbol{E}_{\mathrm{HT}}=-\boldsymbol{V}_{\mathrm{HT}} \times \boldsymbol{B}$, the ratio $D / D_{0}$ expressing the fraction of $\boldsymbol{E}_{i}$ remained un-transformed in the HT frame, and the three components of the HT velocity, $\boldsymbol{V}_{\mathrm{HT}}$, in GSE.

\begin{tabular}{lccccc}
\hline & Time interval & correl. & slope & $D / D_{0}$ & $V_{\text {HT }}[\mathrm{km} / \mathrm{s}]$ \\
\hline $\mathrm{C} 2$ & {$[28.5-41.4]$} & 0.94 & 1.03 & 0.11 & {$[232,58,-102]$} \\
$\mathrm{C} 4$ & {$[27.4-47.3]$} & 0.99 & 1.00 & 0.02 & {$[200,177,-203]$} \\
$\mathrm{C} 3$ & {$[58.9-78.9]$} & 0.99 & 1.00 & 0.02 & {$[77,151,-181]$} \\
$\mathrm{C} 1$ & {$[65.8-81.9]$} & 0.96 & 0.98 & 0.09 & {$[251,156,-150]$} \\
\hline
\end{tabular}

planar solutions imply, in case of C3 and C1, a systematic difference between the two sets of normals. The effect can be also noticed in the third and fourth panels of Figs. 8, 9 and 10. Actually, the analysis presented in Appendix B makes clear that this difference in orientation is due to the different time-intervals on which the two normals are based. In fact, in case of $\mathrm{C} 3$ and $\mathrm{C} 1$, for all centred intervals starting from $\left[T_{c i}-\tau_{i}, T_{c i}+\tau_{i}\right]$ up to the largest one used in the analysis, the planar, interval-specific MVAB normals lie in the range of angular variation for the 2-D, instantaneous normals. This is exactly the result one expects when the planar MVAB technique is applied to an ideal 2-D discontinuity (see Dunlop and Woodward, 1998).

\subsection{Magnetopause normal velocity}

Table 3 presents a comparison between the 2-D, non-planar method and the various planar techniques in terms of MP normal velocity prediction. In the planar case, we show the results obtained by combining the MVAB and HT analyses, those provided by the MFR analysis, by the two variants of the timing analysis, i.e. CTA and CVA, and by assuming a locally planar MP at each pair of consecutive satellites.

For the 24 June 2003 event, reasonably good HT frames at all four Cluster satellites were identified. Table 4 presents the results obtained from this analysis. For a meaningful comparison with the 2-D method, the intervals of analysis (shown on column 2) were chosen as close as possible to the central crossing intervals $\left[T_{c i}-\tau_{i}, T_{c i}+\tau_{i}\right]$. In case of $\mathrm{C} 1, \mathrm{C} 3$ and $\mathrm{C} 4$ the plasma measurements together with the approximation $\boldsymbol{E}=-\boldsymbol{v} \times \boldsymbol{B}$ for the electric field were used, whereas for $\mathrm{C} 2$, where no ion plasma data are available, electric field measurements provided by the EFW instrument were employed.

As Table 3 shows, by following the standard planar procedure, i.e. projecting the HT velocities, $\boldsymbol{V}_{\mathrm{HT}}$, on the normals provided by the planar, nested MVAB analysis, one

tively. 
obtains values for the MP normal velocity significantly different from the C2_OpTA_A solution. In fact, for C4 and C3, i.e. the satellites where the HT frame identification was most successful (see Table 4), these values are negative, which, considering that one deals with an in-bound transition, represents an inconsistency. A possible explanation could be that in the HT analysis one tries to explain the observed electric field as being produced by a time-stationary structure moving with constant velocity, both in direction and magnitude, relative to the point of observation, a situation one can hardly encounter in case of a 2-D MP ${ }^{8}$. The relatively small number of data points on which the HT analysis is based, i.e. five for both $\mathrm{C} 4$ and $\mathrm{C} 3$, could be another explanation.

The MFR method provides individual MP normal velocities, $V_{M F R}$, significantly higher than in the 2-D MP method. We have no particular explanation for this result; a more detailed investigation, like using synthetic data to study how the planar MFR method performs on a 2-D discontinuity, as done by Dunlop and Woodward (1998) for the MVAB technique, could prove to be useful.

The planar CTA and CVA timing techniques provide MP global normal velocities that are $\sim 60 \%$, and, respectively $\sim 75 \%$ higher than in the 2-D MP method (see Tables 3 and 5 from Sect. 6).

The technique that evaluates the normal MP velocity by assuming a locally planar MP at each pair of consecutive satellites, provides results closer to the C2_OpTA_A solution for the first pair of satellites (C2 and C4), i.e. 55.3 compared to $54.2 \mathrm{~km} / \mathrm{s}$, respectively. For the next interval, between the $\mathrm{C} 4$ and $\mathrm{C} 3$ crossing times, the two methods provide significantly different results, i.e. 11.4 and $46.2 \mathrm{~km} / \mathrm{s}$, respectively. That can be explained by the large separation distance between these satellites, when the effects of the MP curvature cannot be neglected. For the last pair of satellites (C3 and C1) the results are also significantly different, i.e. 22.9 and $40.7 \mathrm{~km} / \mathrm{s}$, respectively, in spite of their close separation. The reason for that resides in the difference between the individual MVAB normals, on which the calculation of $V_{\text {pair }}$ is based, and the average geometrical normals based on the 2-D solution, i.e. $\boldsymbol{n}_{2 \mathrm{D}}^{\text {ave }}$ (see the last paragraph of Sect. 5.2).

\section{Global parameters of the 2-D solutions}

Table 5 presents the global values that characterize all six valid solutions obtained by applying different implementations of the 2-D, non-planar method to the analyzed event. Two other solutions, namely P2_TA and C1_TA, had to be

\footnotetext{
${ }^{8}$ One arrives at a similar conclusion when a version of HT analysis that accounts for a possible accelerated motion of the HT frame (see Sonnerup et al., 1987) is used. For example, in case of C3, for the interval indicated in Table 4 ( 5 points) the instantaneous HT velocity projected on the nested MVAB normal is $-13.9,-11.8$, $-9.8,-7.7,-5.6 \mathrm{~km} / \mathrm{s}$, giving an average MP normal velocity of $-9.8 \mathrm{~km} / \mathrm{s}$.
}

abandoned because, although mathematically correct, they do not offer a physical description of the MP parameters and dynamics (see Blagau, 2007).

A close look at the corresponding MP macroscopic parameters (thickness, normal velocity, radius of curvature, magnetic variance) and at the general MP dynamics, yields similarities between these solutions. We therefore classified them in three groups, each indicated by a different color in Table 5. For the solutions belonging to the first group, i.e. for P2_OpTA, C2_TA, and C2_OpTA_A (pink background), the lowest values of the global normal magnetic field variance were obtained but one also needs to limit their range of validity in order to avoid an artificial return of the first pair of satellites to the MP interior. The second group, consisting of the solutions P1_OpTA and C1_OpTA (green background), imply a unidirectional displacement of the MP. The remaining solution, i.e. C2_OpTA_B (blue background), implying two degrees of freedom for the MP movement, is not related with any other. Note that in Sect. 4 we presented one solution from each group.

On the first column of Table 5 we present the angle $\alpha$, between two normals already introduced in Sect. 4 and shown on Figs. 8, 9, and 10, namely the average normal provided by the nested, planar MVAB technique, $\langle\boldsymbol{n}\rangle_{\text {MVAB }}$, and a median MP normal provided by the 2-D solution, pointing to the exterior (in the geometrical sense), $\langle\boldsymbol{n}\rangle_{\mathrm{ext}}$. The parameter in the second column expresses the variation in the orientation of the 2-D instantaneous normals during the crossing intervals, being the average of $\Delta \boldsymbol{n}_{2 \mathrm{D}}$ (see the description of Table 3 from Sect. 5).

The third and fourth columns present the values obtained for the MP radius of curvature and thickness, respectively. Since for a parabolic layer neither its radius of curvature nor its thickness are constant, the "global" values are shown, computed as geometrical mean (for the radius of curvature) or as arithmetical mean (for the thickness) of the corresponding individual quantities determined at each satellite ${ }^{9}$. Based on HIA C3 measurements, the average thermal proton gyroradius for the MP interval is $133 \mathrm{~km}$, and the average proton inertial length is $270 \mathrm{~km}$.

The next column presents the MP global normal velocity, obtained as arithmetical mean of the individual average normal velocity, $\boldsymbol{v}_{2 \mathrm{D}}^{\mathrm{ave}}$. Then the global normal magnetic field variance and global value of $\left\langle B_{n}\right\rangle$ corresponding to the central intervals are shown. As in Table 3 , the blue numbers designate values based only on data from $\mathrm{C} 1, \mathrm{C} 3$ and $\mathrm{C} 4$, considering that the $\mathrm{C} 2$ contribution to these global quantities is

\footnotetext{
${ }^{9}$ Actually, for a parabolic layer the individual values of these quantities vary as well. In our model only the distance between the MP leading and trailing edges along the parabola's axis of symmetry is fixed (see Appendix A). Therefore the MP thickness, measured along the local normal, may vary between the point where the satellite enters the discontinuity and the exit point. For the analyzed event such variations are of the order of a few percent.
} 
Table 5. MP characteristics for the 24 June 2003 event, based on the valid solutions provided by different implementation of the 2-D, nonplanar method. Global values corresponding to MP orientation, radius of curvature, thickness, normal velocity as well as normal magnetic field variance and normal magnetic field component are provided. The last column indicates whether a spatial limitation in the validity range of the corresponding solution is required in order to avoid an artificial return of the first pair of satellites to the MP interior. For the error estimate of the MP radius of curvature, thickness and normal velocity, see Appendix C.

\begin{tabular}{lccccccccc}
\hline & $\begin{array}{c}\alpha \\
{[\mathrm{deg}]}\end{array}$ & $\begin{array}{c}\left\langle\Delta \boldsymbol{n}_{2 \mathrm{D}}\right\rangle \\
{[\mathrm{deg}]}\end{array}$ & $\begin{array}{c}\text { radius } \\
{\left[R_{\mathrm{E}}\right]}\end{array}$ & $\begin{array}{c}\text { thickness } \\
{[\mathrm{km}]}\end{array}$ & $\begin{array}{c}V_{n} \\
{[\mathrm{~km} / \mathrm{s}]}\end{array}$ & $\begin{array}{c}\boldsymbol{B} \text { var. } \\
{[\mathrm{nT}]}\end{array}$ & $\begin{array}{c}\left\langle\mathrm{B}_{\mathrm{n}}\right\rangle \\
{[\mathrm{nT}]}\end{array}$ & $\begin{array}{c}\text { limitation? } \\
{[\text { yes } / \mathrm{no}]}\end{array}$ \\
\hline P1_OpTA & 9.0 & 0.0 & 2.87 & 810 & 52.0 & 3.28 & 2.76 & $-1.5-1.8$ & no \\
\hline P2_OpTA & 7.1 & 6.9 & 1.81 & 765 & 49.7 & 2.762 .52 & $-1.0-1.4$ & yes \\
C2_TA & 3.9 & 6.4 & 1.85 & 740 & 47.5 & 2.502 .26 & $-0.7-1.1$ & yes \\
C1_OpTA & 9.8 & 2.0 & 2.09 & 824 & 52.8 & 3.22 & 2.85 & $-1.5-1.9$ & no \\
C2_OpTA_A & 3.9 & 6.4 & 1.85 & 740 & 47.5 & 2.502 .26 & $-0.7-1.1$ & yes \\
C2_OpTA_B & 6.7 & 2.2 & 2.39 & 755 & 48.4 & 3.012 .71 & $-1.2-1.6$ & no \\
\hline
\end{tabular}

misleading due to the local magnetic irregularities. A comparison with the planar results can be made by looking at similar quantities from Table 3 .

The last column indicates which solutions need a limitation in their range of validity, in order to avoid an artificial MP crossing (see Fig. 9 and the related discussion).

In Appendix $\mathrm{C}$, an analysis on the errors in the values of MP crossing parameters is presented. The solutions from the first group, i.e. C2_OpTA_A, C2_TA, and P2_OpTA, are examined and compared. By looking on Tables 3 and 5, one can notice that these solutions involve the highest dynamical behavior for the MP, i.e. small radius of curvature, high variations in the instantaneous normal velocity and relatively high variations in the instantaneous, geometrical normal orientation. Therefore, one can presume that their characteristics will exhibit higher variations than in case of other solutions.

The analysis shows that, for the 24 June 2003 event, the $1 \sigma$ uncertainties in the crossing parameters remain in a reasonably limit, typically $\sim 5-11 \%$, with the exception of the radius of curvature that experiences higher variations (see Table C1 from Appendix C). In our opinion, the regular and well resolved MP transition at each satellite, which ensures an accurate timing information determination, largely accounts for this relatively low error level, even if the Cluster two-pairs configuration is not a favorable one. Then, by comparing the parabolic and cylindrical solutions, the analysis indicates smaller errors in the latter case. Also, the combined timing - MVAB analysis produces stabler results than the straight timing analysis.

\section{Discussion of the 2-D method results}

Based on the results obtained from the new method, in this section we discuss the MP dynamics and the presence of a non-zero normal magnetic field component on the 24 June 2003 crossing event.

\subsection{The MP dynamics on 24 June 2003}

All the six valid solutions, obtained when applying the various implementations of the new method to the 24 June 2003 event, indicate a convex shape for the local MP, with radius of curvature between $\sim 1.8$ and $\sim 2.9 R_{\mathrm{E}}$ (see Table 5 ). This result can be compared with the curvature of a model MP at the scale of Cluster inter-spacecraft separation distance. Based on the Roelof and Sibeck (1993) MP model, we found a radius of curvature of about $19.3 R_{\mathrm{E}}$ at the Cluster crossing location. Considering that we analyzed an in-bound transition, it means that the spacecraft encountered a local bulge in the MP.

In the present study we do not concentrate on the physical mechanism that produced the 2-D MP feature encountered by Cluster. However, the large flow shear across the MP ( $350 \mathrm{~km} / \mathrm{s})$ suggests the Kelvin - Helmholtz (KH) mechanism as a possible explanation. In the lower right corner of Fig. 3 from Sect. 2.1 we showed the tangential components of the magnetic field and flow velocity vectors measured in the regions adjacent to the MP. The unit vector $\boldsymbol{k}$ (shown in green in Fig. 3), contained in the tangent plane and perpendicular to the invariant direction, would then be roughly oriented along the wave vector ${ }^{10}$. The data suggest that the wave will not

\footnotetext{
${ }^{10}$ One can notice that $\boldsymbol{k}$ is oriented between the direction perpendicular to $B_{\mathrm{EXT}}$ and the direction of $V_{\mathrm{EXT}}$, i.e. the magnetic field and the flow velocity in the magnetosheath (black arrows in Fig. 3), making with them an angle of $\sim 19^{\circ}$ and $\sim 22^{\circ}$, respectively. This adds support to the interpretation of a wave driven by the magnetosheath flow, propagating on the MP surface almost perpendicular to the magnetic field, which, from energy considerations, is the most favored direction.
} 
grow into the nonlinear regime, since for all directions in the plane perpendicular to the invariant direction, the $\mathrm{KH}$ instability criterion (see Chandrasekhar, 1961) is not satisfied.

If Cluster encounters a MP surface wave of KH origin (or produced by any other mechanism, e.g. solar wind pressure changes), the method developed in this paper can be used to estimate its parameters, like the amplitude, wavelength, and periodicity. The implementation most appropriate to describe the physical situation would be that of a parabolic MP having 2 degrees of freedom, one for the inward-outward motion and one for the tangential propagation. Note that for surface normals close to the parabola axis of symentry, the chosen model is a good approximation for a traveling sine wave. The condition is fulfilled for the analyzed event, where the corresponding P2_OpTA solution provides an angle between the surface normals' average orientation, $\langle\boldsymbol{n}\rangle_{\mathrm{ext}}$, and the parabola's axis of symmetry of $\sim 24^{\circ}$.

We determined the parameters of a sinusoidal surface wave that best fits with the results provided by the P2_OpTA implementation in the following way: through the points where the Cluster satellites encounter the corresponding parabolic layer, we fitted a sinusoidal curve, obtaining an amplitude of $\sim 1.7 R_{\mathrm{E}}$, and a wavelength of $\sim 8.9 R_{\mathrm{E}}$. Considering that the P2_OpTA solution predicts a MP tangential velocity of $\sim 77.9 \mathrm{~km} / \mathrm{s}$, one arrives at a periodicity of $\sim 12 \mathrm{~min}$. The result we obtained is in accordance with other published results, reporting on MP surface wave observations (see for example Kivelson and Chen, 1995; Foullon et al., 2008, and references therin).

In principle, in the new method one could have chosen a sinusoidal shape layer to model the MP, with the advantage of estimating directly the amplitude and wavelength of a traveling surface wave. However, instead of this direct approach we preferred to start with the development of the parabolic model for both methodological and physical reasons. Firstly, for a sinusoidal shape one has to resort to numerical methods, where bigger difficulties are expected in finding and sorting solutions that, although mathematically correct, are not acceptable from physical perspective. By contrast, for the parabolic model - which is the first approximation for the sinusoidal shape, provided that the resulting surface normals are close to the parabola axis of symmetry - we were able to find an algebraic, i.e. exact solution. The MP macroscopic parameters and the corresponding error estimates obtained in this way, i.e. through a robust and simple algorithm, have the important advantage of providing a reference, useful when evaluating the results from other models, where the solution can be obtained only numerically. Secondly, a parabolic profile is characterized by one parameter, i.e. the quadratic coefficient $a$ (like in Eq. A2), whereas two parameters are needed to characterize a profile of sinusoidal shape, i.e. the amplitude and the wavelength. Since the total number of unknowns that can be determined with the new method is fixed (to 8), more parameters are thus available to characterize the MP dynamics in the parabolic model; taking into account the observations reported in the literature (e.g. Dunlop et al., 2001, 2002), that supports the idea of a rapidly changing MP dynamics, this means a more accurate description of the physical situation ${ }^{11}$.

From an easiness of treatment perspective, the cylindrical case is only slightly more complex than the parabolic one. In order to find a solution one needs to resort to numerical algorithms but, like in the parabolic model, the cylindrical profile is still characterized by one parameter, i.e. the radius of curvature, with the advantage of a more accurate description for MP motion. Note that in the cylindrical model the layer thickness is indeed constant, whereas for other shapes this quantity can vary (see Footnote 9) and one has to check whether the variation is small or not.

\subsection{The normal magnetic field component on 24 June 2003}

As can be seen from Table 5 presented in Sect. 6, all the valid 2-D solutions obtained in the analyzed case imply a non-zero negative value for the global $\left\langle B_{n}\right\rangle$ quantity. Actually, if $\mathrm{C} 2$ is left aside (see the discussion from Sect. 5.2), all the valid 2$\mathrm{D}$ solutions provide values between $-2.0 \mathrm{nT}$ and $-1.0 \mathrm{nT}$ for the individual $\left\langle B_{n}\right\rangle$ quantities (for the C2_OpTA_A solution, these values are presented in Table 3 from Sect. 5).

While one can imagine that the 2-D solutions could introduce a non-realistic offset in the values of $\left\langle B_{n}\right\rangle$, we think this is not the case for the 24 June 2003 event. In this respect, for the C2_OpTA_A solution we show in Appendix B that, for all satellites, the $\left\langle B_{n}\right\rangle$ values based on the 2-D, instantaneous normals are actually smaller (in the absolute sense) than the $\left\langle B_{n}\right\rangle$ values based on the planar, interval-specific normals, well beyond the validity range of the 2-D solution.

Also, with the error calculation procedure presented in Appendix C, the estimated standard deviations for the $\left\langle B_{n}\right\rangle$ quantities are relatively small. As an example, for the C2_OpTA_A solution these standard deviations are (in $\mathrm{nT}$ ) $\sim 0.6,0.4,0.1$, and 0.1 for $\mathrm{C} 2, \mathrm{C} 4, \mathrm{C} 3$, and $\mathrm{C} 1$ respectively.

We cross-checked the existence of a non-zero normal magnetic field component by performing Walén tests (see for example Paschmann and Sonnerup, 2008) on the MP intervals $^{12}$. With the Walén test one checks whether the plasma flows at the local Alfvén speed in the HT reference frame. This property defines a rotational discontinuity (RD), implying $\left\langle B_{n}\right\rangle \neq 0$, thought to be caused as a consequence of magnetic reconnection, and encountered by the satellite far from the reconnection site. Although the test is derived based on a planar geometry, one can still presume its ability to correctly

\footnotetext{
${ }^{11}$ For example, in the straight timing analysis, only two parameters are left to describe the motion of a sinusoidal shape layer, i.e. a constant velocity along the primary direction and one along the secondary direction.

${ }^{12}$ For that purpose, we chose intervals of analysis situated more on the magnetosheath side, according to the customary procedure used when identifying rotational discontinuities at the MP.
} 
Table 6. Results from the Walén analysis performed on C4, C3 and $\mathrm{C} 1$ data. For each satellite we show the interval of analysis, the correlation coefficient and regression line slope provided by the HT analysis, and the correlation coefficient and regression line slope obtained in the actual Walén test.

\begin{tabular}{|c|c|c|c|c|c|}
\hline & Time interval & $\begin{array}{r}\mathrm{H} \\
\text { correl. }\end{array}$ & $\begin{array}{l}T \\
\text { slope }\end{array}$ & $\begin{array}{l}\text { Wa } \\
\text { correl. }\end{array}$ & $\begin{array}{l}\text { lén } \\
\text { slope }\end{array}$ \\
\hline $\mathrm{C} 4$ & [07:36:25-07:36:38] & 1.00 & 1.00 & 0.97 & 0.51 \\
\hline $\mathrm{C} 3$ & [07:36:52-07:37:17] & 0.99 & 1.01 & 0.93 & 0.45 \\
\hline $\mathrm{C} 1$ & [07:36:55-07:37:12] & 0.96 & 1.05 & 0.91 & 0.83 \\
\hline
\end{tabular}

identify a RD in the non-planar case, provided that the discontinuity radius of curvature is sufficiently large compared with the kinetic parameters. The condition is fulfilled in the analyzed case where the average proton gyro-radius is two order of magnitude smaller than the MP radius of curvature.

The results are presented in Table 6 and show a positive identification of an RD by $\mathrm{C} 1$, where the correlation coefficient and the regression line between the plasma flow in the HT frame and the local Alfvén velocity are close to the ideal value of 1 . For this satellite, the strong correlation in the MP region between the magnetic field change and the bulk velocity change is evident from panels 4 and 6 in Fig. 2. For C3 and C4, where a closer analysis clearly indicates a large dynamic variation in the plasma velocity components specific to an RD, we evaluate the results as being marginally supportive. The negative values of $\left\langle B_{n}\right\rangle$ indicated by all 2$\mathrm{D}$ solutions, meaning a magnetic field component oriented into the magnetosphere, are also consistent with the positive slopes obtained in the Walén tests.

The accuracy of the method presented in this article to detect, in general, the presence of non-zero magnetic field component along the MP normal, deserves further investigations, on synthetic data as well as on other real cases. Nevertheless, there are two aspects that lend credence on its results. Firstly, in the new method one can use simultaneously and self-consistently the timing information and the $\nabla \cdot \boldsymbol{B}=0$ conservation law, obtaining solutions that comply with the requirement of minimum normal magnetic field variance. Secondly, in the new method the data from all four satellites are simultaneously participating in the analysis and the determined MP normal directions and normal velocities are linked all the time through the geometry and through the motion provided by the solution. This implies a more realistic description for the MP orientation and motion compared to the case of single-spacecraft techniques, where the estimated individual MP normals and normal velocities are, by definition, decoupled one from each other.

In Sect. 5.2 this characteristic of the 2-D solution helped us to establish that local magnetic irregularities (magnetic islands, noise etc.) play an important role in case of $\mathrm{C} 2$, affecting the results of the MVAB technique when applied on the central interval. We mention here that Dunlop and Woodward (1999), when studying, with synthetic data, how the local normal direction of a non-planar, ideal discontinuity, can be obtained by employing the planar MVAB method, showed that the errors are smaller when points from a short interval around the center of the structure are used. However, in case of a real event, a situation similar to that encountered by $\mathrm{C} 2$ on 24 June 2003 may occur.

The fact that we relied in Sect. 2.1 on the results provided by the constrained MVAB analysis may look like an inconsistency with the presence of a non-zero normal magnetic field component. However, for a discontinuity like the MP, having only a small or no perpendicular magnetic field component, the error in estimating its normal with MVAB is probably smaller when the constraint $\left(\left\langle B_{n}\right\rangle=0\right)$ is used (see for example Blagau, 2007). Another aspect is whether the presence of reconnection, with its specific wedge-like geometry, interferes with our assumption of constant thickness for the MP. However, far from the reconnection site, where the observations are made, it is reasonably to neglect any such influence at the scale of inter-spacecraft separation distance.

\section{Summary and general discussions}

In this article we extended the four-point timing method used for determining the macroscopic parameters of a planar MP, in order to accommodate the situations when the discontinuity behaves like a 2-D, non-planar layer on the scale of the inter-spacecraft separation distance. The MP was locally modelled either as a parabolic or as a cylindrical layer of constant thickness, with one or two degrees of freedom for the motion in the plane perpendicular to the invariant direction.

The MP local parameters (orientation, motion, thickness, radius of curvature) are obtained simultaneously from a system of eight equations, utilizing the satellites encounter of the MP leading and trailing edges according to the timing information extracted from the data. There are actually two techniques that we developed. The first relies on the timing information alone, being a straight extension of the planar CTA technique to the 2-D geometry. In the second technique one exploits the fact that, according to the model, the MP primary direction of movement is completely prescribed by one angle. This allows us to use self-consistently the timing information and the $\nabla \cdot \boldsymbol{B}=0$ conservation law, obtaining solutions that comply with the requirement of minimum normal magnetic field variance. The need of an additional assumption, proposed in earlier work (Mottez and Chanteur, 1994; Dunlop and Woodward, 1998), i.e. that of negligible effects due to non-planarity on the MVAB results, is thus avoided.

To illustrate the new method, we selected one MP transition observed by Cluster on 24 June 2003. For this event the planar MVAB and MFR methods provide individual normals having different orientation, but all of them being close to one plane. This was interpreted as Cluster encountering a 
2-D, non-planar MP, with the invariant direction oriented perpendicular to that plane. The results from the standard, planar timing analysis, providing MP normals well apart from the individual MVAB and MFR normals, and from the HT analysis, providing MP normal velocities at $\mathrm{C} 3$ and $\mathrm{C} 4$ locations inconsistent with an in-bound crossing, add support to the non-planar assumption.

When the different implementations of the new method (parabolic or cylindrical geometry, one or two degrees of freedom, straight timing analysis or combined, timingMVAB analysis) are applied to the 24 June 2003 event, one obtains six valid solutions. The solutions are consistent and stable, indicating a convex shape for the local MP. The global values of the parameters defining these solutions are presented in Table 5 from Sect. 6. In spite of the Cluster two-pairs configuration in this event, the analysis from Appendix $\mathrm{C}$ indicates that the corresponding errors are relatively small, e.g. $\sim 5-11 \%$ for the MP normal velocity. One expects an even smaller level of errors for events when the spacecraft configuration is closer to that of a tetraheadron.

In Sect. 5 we chose one particular 2-D solution, i.e. C2_OpTA_A, for an extensive comparison with the planar results. We showed that, for the central intervals, i.e. for its range of validity, the C2_OpTA_A solution provides a smaller global magnetic field variance along the 2-D, instantaneous normals than the planar MVAB method. For other 2-D solutions, depending on the particular implementation, the global normal magnetic field variance on the central intervals is lower or approximately the same as in the planar case (see Tables 5 and 3). The same quantity computed according to the standard planar method, i.e. using nested intervals and normals provided by the nested MVAB analysis, is systematically higher than in the case of the 2-D solutions ${ }^{13}$.

The method developed in this article provides a means to quantitatively determine the geometry and motion of a 2-D, non-planar discontinuity. We illustrated this by discussing the MP dynamics and the presence of a non-zero normal magnetic field component on the 24 June 2003 crossing event, i.e. the aspects, mentioned in Sect. 1, that motivate the effort of finding the MP macroscopic parameters.

Depending on the event, applying the standard, planar methods to estimate the normal motion of a non-planar 2-D discontinuity, may provide results significantly different than those of the new method (for the analyzed event, see Table 3 and the discussions from Sect. 5.3). Correspondingly, the

\footnotetext{
${ }^{13} \mathrm{~A}$ similar finding is obtained by comparing the global Faraday residues, namely lower values for this quantity based on the instantaneous MP normals and normal velocities provided by the 2-D solutions than in the planar case (see Blagau, 2007). Although this result is consistent with the other results reported in this article and could represent an independent way of proving that the 2-D method provides a better identification of the MP orientation and motion, due to the uncertainties in the electric field data for this event we decided not to use it as an argument.
}

same situation is present when the discontinuity thickness is estimated.

The method shown here represents a logical extention of the techniques described in Sect. 1, seeking to determine the local parameters of a discontinuity in a single, individual crossing event. A different line of approach for investigating a boundary region is that of the reconstruction methods, i.e. the methods of empirical reconstruction (e.g de Keyser, 2008) and of Grad-Shafranov (GS) and MHD reconstructions, (e.g Sonnerup et al., 2008; Hasegawa et al., 2005). In the empirical reconstruction method the position of the boundary is tracked by converting a time series of in-situ observations into spatial information; spatial profiles of physical quantities whithin the boundary are thus obtained. With the GS and MHD reconstructions, maps of fields and plasma quantities are created in a region surrounding the spacecraft path by integrating the GS or MHD equations. A main assumption common to the reconstruction methods is that all the observed time variability is due solely to the convection of a fixed structure past the spacecraft position. While the reconstruction methods aim at much more than finding the macroscopic parameters of a discontinuity, below we enumerate some aspects in favor of our method if the main goal is the analysis of the macroscopic parameters:

- the reconstruction techniques imply evolved techniques of optimization and/or integration, which requires considerable attention and skill when applied. On the contrary, our method being simpler, offers more control when is used.

- the conditions for applying the reconstruction methods include that of no intrinsic temporal change in the structure of the boundary. Also, in the GS and MHD reconstructions, the existence of a proper frame (usually the HT frame) and the validity of MHD equations are demanded. Our technique is less sensitive to these requirements, being based on the timing information and on the $\nabla \cdot \boldsymbol{B}=0$ conservation law.

- the reconstruction methods make use of plasma data, i.e. plasma velocity or other higher moments of plasma velocity distribution function, usually measured with less accuracy than the magnetic field. For example, in the empirical reconstruction the boundary normal velocity is obtained by an optimization procedure so that the measured plasma velocity along an average normal direction is matched and a spatial profile fits other physical observable.

- in the empirical reconstruction, the obtained spatial profiles represent an average structure over the whole interval of analysis, which typically includes many transitions, not an individual boundary crossing. In the 2D reconstruction, (e.g. de Keyser and Roth, 2003), the tangential velocity is not directly determined but is assumed from outside. 
The central assumption adopted in this paper, namely that the MP has a constant thickness, certainly introduces a limitation but also leads to a significant simplification of the task to infer the discontinuity parameters. We based this assumption on the arguments presented in Sect. 1 and on the results reported for the planar case (Haaland et al., 2004; Blagau, 2007).

In general, limitations in the validity range of any solution provided by the new method result from the fact that they describe the MP boundary only locally. In Table 5 from Sect. 6, we indicated the solutions when such a limitation was needed, to avoid a re-encounter of the MP by the first pair of satellites ( $\mathrm{C} 2$ and $\mathrm{C} 4)$ at the end of the event. This aspect could be in fact a consequence of another limitation introduced by the method, namely the shape itself we assumed for the MP layer; one could recall here observations reporting on the MP surface waves having a steeper leading (e.g. Owen et al., 2004) or trailing (e.g. Kivelson and Chen, 1995) edge. However, since in the present paper we only intend to demonstrate the capability of the new tool, we do not enter into this controversy; other reasons for adopting a parabolic or a cylindrical shape were discussed in Sect. 7.1.

The many facets of the new technique were illustrated by treating in details one MP crossing observed by Cluster. A continuation of the present work is therefore needed both to show more clearly the capabilities of the method and to investigate its possible developments. Below we present a list of topics to be addressed in the future.

- the investigation of other events. By comparing the results obtained under different conditions one can discriminate between the general characteristics of the method and what is specific to each particular event. It will also be useful in evaluating how valid the constant thickness assumption is.

- as explained in Sect. 3, in this article we combined the timing analysis with the MVAB technique. In principle one can combine in a similar way the timing analysis with other techniques, like for example MFR. Furthermore, one can use simultaneously both MVAB and MFR, by adding the global magnetic field variance and the global Faraday residues and search for the direction of MP movement that minimizes this quantity (a procedure to combine variances of different quantities is described in Sonnerup et al., 2006). Finding a MP crossing to illustrate this procedure would be instructive.

- an investigation of method performance using synthetic data would allow to study the dependence of errors in predicting the discontinuity macroscopic parameters on different spacecraft configurations. It would also allow to study how the planar MFR method performs on a 2$\mathrm{D}$, non-planar discontinuity (see the observation made in Sect. 5.3)
- in Sect. 7.1 we discussed the rationale for using the parabolic and the cylindrical profiles to represent the MP layer. One could investigate the possibility to model the MP in other ways, like as a sinusoidal shape mentioned there, or as a shape having a steeper leading or trailing edge. In that case, finding valid solutions could benefit from the results based on the two geometries presented in this article.

- the new method was designed to be applied when the boundary acquired a 2-D shape. For a planar discontinuity, the use of standard, planar techniques would ideally produce parallel normal directions. However, when these normals are not exactly parallel, one may think that the method presented here can be adjusted to the planar situation, e.g. by optimizing the direction of the planar timing normal so that the global normal magnetic field variance is minimized.

\section{Appendix A}

\section{Details of the 2-D method}

This appendix provides details about the models and the techniques employed in the article for determining the macroscopic parameters of a 2-D, non-planar discontinuity. For the complete report about how the actual solutions were obtained in each particular implementation of the method, the reader is referred to Blagau (2007).

Below we consider that the timing information, i.e. the times when each satellite detects the MP leading and trailing edges, and the orientation of the invariant direction $l$, have been extracted from the data. The satellites can then be ordered according to the times when they detect the MP leading edge. In what follows by satellite 1 one understands the first spacecraft that encounters that surface, by satellite 2 the second one and so on. The origin of time corresponds to the instant when the MP leading edge is detected by the first satellite. The satellites positions are regarded as fixed points in space, an assumption well-justified considering their small orbital velocity relative to the typical MP velocity. For example, for the 24 June 2003 event, the satellites orbital velocities along the MP normal were $\sim 0.11 \mathrm{~km} / \mathrm{s}$, to be compared with the average MP normal velocity of $\sim 50 \mathrm{~km} / \mathrm{s}$ (see Table 5).

We will work in the fixed orthogonal reference frame $(\boldsymbol{x}, \boldsymbol{y}, \boldsymbol{l})$, centred on the first satellite and oriented with the third axis along the MP invariant direction (see Figs. 6 and 7 from Sect. 3, referring to the parabolic and cylindrical models, respectively). The $\boldsymbol{y}$ axis, lying in the plane of the normals, is oriented along what we called the primary direction. As mentioned in Sect. 3, the method assumes either one or two degrees of freedom for the MP displacements. In the former case, by primary direction one understands the only possible direction allowed for the MP movement. In the latter 
case, when the MP has a compound motion along two mutually perpendicular directions, the primary direction is the direction along which the model allows for a variable velocity, therefore implying an acceleration or even higher terms in the velocity change. Along the other, i.e. $\boldsymbol{x}$, direction, called the secondary direction, the MP is allowed to move with a constant velocity.

We will use the following notations (see also Figs. 6 and 7 from Sect. 3):

- the primary direction of MP motion is specified by the angle $\beta$, between $\boldsymbol{y}$ and $\boldsymbol{m}=\boldsymbol{R}_{2} \times \boldsymbol{l}$ (here $\boldsymbol{R}_{2}$ designates the position vector of the second satellite relative to the first satellite). Note that the direction of $\boldsymbol{m}$, chosen as reference direction in the plane of the normals, can be completely established for each event.

- $A_{0}, A_{1} \ldots A_{N}$ are the polynomial coefficients that describe the MP primary velocity, i.e. along the $\boldsymbol{y}$ direction: $U(t)=A_{0}+A_{1} t+\ldots+A_{N} t^{N}$.

- $v$ is the speed along $\boldsymbol{x}$ direction, when a second degree of freedom for the MP layer is allowed.

$-d$ is the magnetopause half-thickness.

- $s$ and $C$ designate the initial position of the MP, i.e. at $T_{c 1}-\tau_{1} \equiv 0$. More precisely, in case of the parabolic layer the parameters refer to the $x$ and, respectively, $y$ coordinate of the leading edge apex location, whereas in case of the cylindrical layer they designate the coordinates of the center of curvature.

- the spatial scale of the structure is described in the parabolic model by the quadratic parameter $a$. For example, at the initial moment the parabola has the equation: $y(x)=C+a(x-s)^{2}$. In case of the cylindrical layer model, the corresponding parameter is the radius of curvature, $R$, of the inner MP surface.

\section{A1 The parabolic layer model}

Figure 6 from Sect. 3 shows a parabolic MP cross-section at successive times, when its edges encounter the Cluster satellites (situated at origin and at the positions indicated by the $\boldsymbol{R}_{2}, \boldsymbol{R}_{3}$ and $\boldsymbol{R}_{4}$ vectors). The invariant direction $\boldsymbol{l}$ is pointing into the paper. According to the proposed model, the direction of primary movement $\boldsymbol{y}$ is along the (common) axes of the parabolas that define the MP leading and trailing surfaces. By "constant thickness" of the layer one understands that the distance along $\boldsymbol{y}$ between the leading and trailing edges is the same. In Fig. 6 we have chosen to represent the situation when the Cluster satellites are initially situated inside the opening made by the parabola's branches and when also a tangential velocity is present along the secondary direction $\boldsymbol{x}$. For one particular event, the initial location of the spacecraft (inside or outside the parabola's branches) and the secondary motion (in the positive or negative $\boldsymbol{x}$ direction) depend on the actual timing information and on the Cluster configuration, resulting directly from the equations described below.

In order to determine the MP macroscopic parameters, one imposes the conditions that, during its motion, the margins of the parabolic layer encounter the satellites positions at the proper times, i.e. four conditions for encountering the leading MP edge at $T_{c i}-\tau_{i}$ and four conditions for encountering the trailing MP edge at $T_{c i}+\tau_{i}$. Mathematically, these conditions can be formulated, respectively, as

$a\left(X_{i}-X_{a p}\left[T_{c i}-\tau_{i}\right]\right)^{2}=Y_{i}-Y_{a p}\left[T_{c i}-\tau_{i}\right]$

$a\left(X_{i}-X_{a p}\left[T_{c i}+\tau_{i}\right]\right)^{2}=Y_{i}-Y_{a p}\left[T_{c i}+\tau_{i}\right]+2 d$

with $i=1 \ldots 4$. In the above equations $\left(X_{i}, Y_{i}\right)$ designate the coordinates of satellite $i$, while $\left(X_{a p}[t], Y_{a p}[t]\right)$ refer to the leading edge apex position, which varies in time:

$X_{a p}[t]=s+v t$

$Y_{a p}[t]=C+\sum_{i=0}^{N} t^{i+1} A_{i} /(i+1)$

The quantities $a, d, s$, and $C$ have to be obtained from the system of eight equations (A1) and (A2). The other four unknowns are described below.

\section{A1.1 Straight timing analysis}

In the straight timing analysis technique one does not know from the beginning how $\boldsymbol{x}$ and $\boldsymbol{y}$ are oriented in the plane of the normals, i.e. the angle $\beta$ is an unknown to be determined by solving the conditions (A1) and (A2).

It can be shown that one needs a MP having two degrees of freedom in this implementation of the parabolic model. Therefore, the remaining unknowns, describing the MP velocity, are $A_{0}$ and $A_{1}$ for the primary motion and $v$ for the velocity along the secondary direction. A unique algebraic solution with physical significance (i.e. with a positive value for $d$ ) has been found in this case.

\section{A1.2 Combined timing - MVAB analysis}

In the combined timing - MVAB analysis algorithm, the angle $\beta$ is an input parameter and therefore one additional unknown can be used to characterize the MP movement. In these conditions, a third coefficient, $A_{2}$, to describe the velocity along the primary direction $\boldsymbol{y}$ can be added, or one can suppress the movement along the secondary direction and use four coefficients for the velocity along $\boldsymbol{y}$. Note that now the quantities $X_{i}$ and $Y_{i}$, designating the position of the satellites in the working reference frame $(\boldsymbol{x}, \boldsymbol{y}, \boldsymbol{l})$, are known.

In either case it can be shown that to each direction (each angle $\beta$ that determines the values of $X_{i}$ and $Y_{i}$ ) corresponds one solution. One can then readily compute a set of quantities that characterize the MP orientation and motion, like for example the instantaneous surface normal at each satellite 
position. The algorithm developed in this implementation changes $\beta$ in steps between $-\pi$ and $\pi$, computes the global magnetic field variance along the instantaneous normals for each corresponding solution (as described in Sect. 3; see also the next paragraphs) and finally selects that direction of MP movement that minimizes this quantity.

To compute the global value of the normal magnetic field variance, one can use the following weighting factors

$p_{i}=\mathbf{T r}_{c i}^{-1}(\lambda) / \sum_{k=1}^{k=4} \mathbf{T r}_{c k}^{-1}(\lambda) \quad i=1 \ldots 4$

where by $\mathbf{T r}_{c i}$ one designates the trace of the constrained, to $\boldsymbol{l} \cdot \boldsymbol{n}=0$, magnetic field variance matrix corresponding to satellite $i$ (references about the variance matrix can be found in Sonnerup and Scheible 1998; Sonnerup et al. 2006). The constraint $\boldsymbol{l} \cdot \boldsymbol{n}=0$ adopted in Eq. (A5) is consistent with the fact that the instantaneous normals are all perpendicular to the invariant direction $\boldsymbol{l}$. For the 24 June 2003 event, the values of $\mathbf{T r}_{c i}$ (in $[n T]^{2}$ ) are 57.9, 45.3, 60.0, and 46.8 for $\mathrm{C} 2, \mathrm{C} 4, \mathrm{C} 3$, and $\mathrm{C} 1$, respectively, leading to the values of $p_{i}$ shown in Table 3.

The main idea behind choosing this definition for $p_{i}$ was that the magnetic field measured at one satellite could be, by whatever reason, more perturbed than at the other, resulting in a higher value for $\mathbf{T r}_{c i}$. In this case its contribution to the global magnetic variance should be diminished. However, as pointed out by Sonnerup et al. (2006), there is no unique way to define the weighting factors.

\section{A2 The cylindrical layer model}

In this model $s$ and $C$ designate the initial $\boldsymbol{x}$ and $\boldsymbol{y}$ coordinates of the layer's center of curvature P (see Fig. 7 from Sect. 3). Another difference is that, instead of the quadratic parameter $a$ from the parabolic model, the spatial scale of the structure is described by the radius of curvature, $R$, of the inner MP surface.

Conditions similar to Eqs. (A1) and (A2) can be formulated for the MP leading and trailing edges, respectively,

$$
\begin{gathered}
\left(X_{i}-X_{\mathrm{P}}\left[T_{c i}-\tau_{i}\right]\right)^{2}+\left(Y_{i}-Y_{\mathrm{P}}\left[T_{c i}-\tau_{i}\right]\right)^{2}=R^{2} \\
\left(X_{i}-X_{\mathrm{P}}\left[T_{c i}+\tau_{i}\right]\right)^{2}+\left(Y_{i}-Y_{\mathrm{P}}\left[T_{c i}+\tau_{i}\right]\right)^{2} \\
=(R+2 d)^{2}
\end{gathered}
$$

with $i=1 \ldots 4$. Here $\left(X_{\mathrm{P}}[t], Y_{\mathrm{P}}[t]\right)$ designate the coordinates of the center of curvature $P$, which vary in time as in Eqs. (A3) and (A4).

In the above equations it is assumed implicitly that the satellites and the MP center of curvature are initially on the same side of the discontinuity, i.e. the first encounter is with the surface having the smaller radius of curvature, $R$. This situation is depicted also in Fig. 7 from Sect. 3. It will become clear from below that this presumption affects only the last part of the following argumentation, because the algorithm finds first all the other unknowns, apart from $R$ and $d$, in a way independent of that. At the end, both possibilities are tested (satellites and the center of curvature located initially on the same side or on opposite sides of the MP) rejecting the one which implies a negative value for $d$.

For finding solutions in the cylindrical model case, numerical methods have been used. Because such methods work more efficient when the number of equations is small, in all sub-cases of the model (straight timing analysis or combined timing - MVAB analysis, one or two degrees of freedom for the MP movement), by expressing some of the unknowns as a function of the others, the initial system of Eqs. (A6) and (A7) has been reduced to a system of only four equations. More specific, in case of the straight timing analysis one arrives at a set of equations in $\beta, A_{i}$, and $v$ (when the second degree of freedom in the MP movement is enabled). In case of the combined timing - MVAB analysis, when the angle $\beta$ is an input parameter, the reduced set of equations are in $A_{i}$ and, possibly, $v$. The reason for keeping these variables is that one has to provide an initial guess for the unknowns and these quantities seemed for us more suitable in this respect. For example the angle $\beta$ has a limited range of variation, i.e. from $-\pi$ to $\pi$, and one could search for the solutions in a loop that varies the initial value for $\beta$ in this range with a step of a few degrees. Also, for the MP velocity one has a better initial guess than, say, for the position of the MP center of curvature.

The entire procedure followed to reduce the initial system of eight equations to one having only four equations is too lengthy to be reproduced here. However, the initial step was to subtract the equations describing the first satellite in Eqs. (A6) and (A7) from the equations referring to the other three satellites, eliminating thus the unknowns $R$ and $d$. From this point on, each sub-case of the cylindrical model differs in the way the variables $s$ and $C$ are expressed as function of the remaining four. Once a numerical solution for $A_{i}$ and, depending on the sub-case, $\beta$ and/or $v$ is found, the values for $s$ and $C$ can be derived as well. Finally, $R$ and $d$ are obtained from the equations corresponding to the first satellite. If the value for $d$ is negative it means that one has to make the transformations $R \longmapsto R+2 d$ and $R+2 d \longmapsto R$ in the initial set of conditions (A6) and (A7), respectively.

\section{Appendix B}

\section{Details of the comparison from Sect. 5}

In this appendix, using different intervals of analysis, we compare the performance of the planar MVAB method and of the C2_OpTA_A solution, in terms of the normal magnetic field variance, average normal magnetic field component $\left\langle B_{n}\right\rangle$, and normal orientation. The investigation is carried out for intervals centred on $T_{c i}$ that progressively increase in size. Starting from a half-width of $\tau_{i}-0.8 \mathrm{~s}$, each new interval is obtained from the preceeding one by adding 
two magnetic field data-points (of $0.4 \mathrm{~s}$ time-resolution), one to the right and one to the left. The largest centred interval used in the analysis does not exceed the largest interval used in the nested MVAB analysis.

Starting from the top, panels 1 to 4 on the left column of Figs. B1 refer to C2, C4, C3 and C1, respectively, and show how the normal magnetic field variance (solid lines) and average normal magnetic field component $\left\langle B_{n}\right\rangle$ (dashed lines) change with the half-width of the interval of analysis. On the abscissas, the interval's half-width is shown, in seconds from $\tau_{i}$, and therefore the origin of these axes corresponds to the central intervals $\left[T c_{i}-\tau_{i}, T c_{i}+\tau_{i}\right]$.

Three sets of normals have been used to compute the plotted quantities. In panels 1 to 4 , the orange lines present for each interval half-width the normal magnetic field variance and $\left\langle B_{n}\right\rangle$ based on normals provided by a constrained MVAB analysis performed for that particular interval. By "constrained" one understands here the condition $\boldsymbol{l} \cdot \boldsymbol{n}=0$. Note that these normals, called hereafter the planar, intervalspecific $M V A B$ normals, are changing with the interval of analysis according to the MVAB theory. The light blue lines designate the evolution of the same quantities based on the individual, planar MVAB normals obtained from the nested MVAB analysis ${ }^{14}$. Because the directions of these normals are fixed in space, in this appendix we will refer to them as the fixed, planar MVAB normals. Finally, in each panel, using the mission color-code, the normal magnetic field variance and $\left\langle B_{n}\right\rangle$ along the 2-D, instantaneous, geometrical normals are shown. Of course, since the C2_OpTA_A solution is valid, strictly speaking, only for the transition intervals $\left[T_{c i}-\tau_{i}, T_{c i}+\tau_{i}\right]$, the above mentioned quantities were computed by extending its validity to larger intervals.

One notes that the magnetic field variance curves based on the planar, interval-specific MVAB normals (solid orange lines) lie always below the similar curves based on the fixed, planar MVAB normals (solid light blue lines). This is not surprising, since the former correspond to the lowest possible magnetic field variance for each interval. At the same time, as expected, all curves referring to the same quantities (magnetic field variance or $\left\langle B_{n}\right\rangle$ ) but based on the two sets of planar MVAB normals, converge as the interval of analysis increases (see the solid and dashed lines colored in orange and light blue).

In panel 5 from the left column, curves representing the "global" magnetic field variances, obtained as weighted sums of the individual normal magnetic field variances, are shown. For computing them, the weighting factors provided by Eq. (A5) from Appendix A and indicated at the bottom of Table 3 have been used. The curves show that even for central intervals having the half-width as large as $\sim \tau_{i}+5 \mathrm{~s}$, i.e.

\footnotetext{
${ }^{14}$ To be more precise, we used their projection on the plane perpendicular to $\boldsymbol{l}$, but giving the fact that the normals lie very close to that plane, taking their projections brings practically no change in the present discussion.
}

well beyond the validity range of the 2-D solution, the global magnetic field variance based on the $2-D$, instantaneous normals (magenta line) is smaller than the same quantity based on the planar, interval-specific normals (orange line), which are the 'best' normals obtained in the planar assumption, implying the minimum magnetic field variance (the global magnetic field variance based on the fixed, planar MVAB normals is shown by the light blue line). In fact, similar statements can be made at the individual level for the satellites C3 (panel 3; compare the satellite specific colored line and the orange line) and C1 (panel 4), while in case of C4 (panel 2) the two curves are very close to each other. The situation is reversed on C2 (panel 1), although the curves are still very close; as discussed later in this appendix, here the local magnetic irregularities play an important role. Considering the above arguments, one can definitely say that, for the above mentioned intervals, the 2-D solution C2_OpTA_A performs better than the planar MVAB solution from the normal magnetic field variance point of view.

In panels 1 to 4 one can notice that the $\left\langle B_{n}\right\rangle$ curves based on the 2-D, instantaneous normals (satellite specific colored dashed line) are closer to the $\boldsymbol{x}$ axis than the similar curves based on the planar, interval-specific normals (yellow dashed line), for most of the intervals with a half-width smaller than $2 \tau_{i}$. This supports the idea that, in the analyzed case, the 2-D solution does not introduce a non-realistic offset in the value of $\left\langle B_{n}\right\rangle$.

The panels 6 to 9 on the right column of Fig. B1 present for each satellite the orientation (in degrees) of the planar, interval-specific $M V A B$ normal (orange crosses), and of the $2-D$, instantaneous normal (the three lines shown in the mission colour code) as function of the interval half-width. The fixed, planar $M V A B$ normal is taken as reference direction (the light blue line at $0^{\circ}$ ). For the $2-D$, instantaneous normal, the plotted lines signify that, during one particular interval of analysis, its orientation changes between the values indicated by dashed lines, with the average orientation $\boldsymbol{n}_{2 \mathrm{D}}^{\text {ave indicated }}$ by the solid line.

For C2 (panel 6) and C1 (panel 9), big variations in the orientation of the planar, interval-specific MVAB normals (orange crosses) can be noticed as the interval half-width becomes smaller than $\tau_{2}$, respectively $\tau_{1}$. This evolution could be associated with the MP rotation and/or with the local, internal (temporal or structural) irregularities. While in general it is difficult to separate between these two factors, for the 24 June 2003 event we think that the local effects dominate. Indeed, as one can see at the beginning of the corresponding traces, the planar, interval-specific $M V A B$ normals change their orientation by more than $2^{\circ}$ from one interval of analysis to the next, i.e. when two magnetic field measurements, of $0.4 \mathrm{~s}$ time-resolution, are successively included in the analysis (one at the right and one at the left of the central time). Supporting this explanation, the corresponding $\left\langle B_{n}\right\rangle$ values (dashed orange line in the panels 1 and 4 on the left column) are relatively big in that regions. 

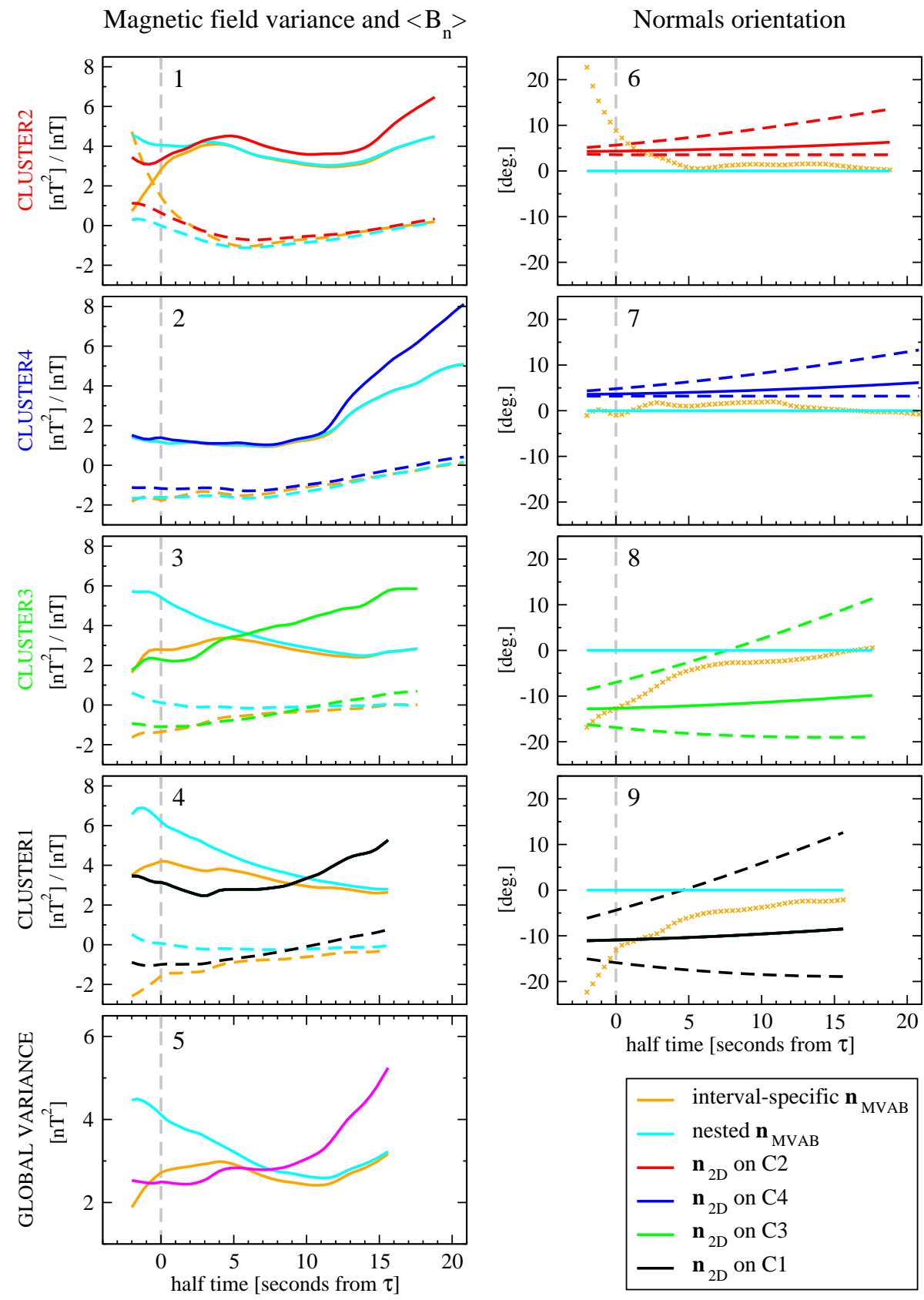

\begin{tabular}{ll|}
- & interval-specific $\mathbf{n}_{\mathrm{MVAB}}$ \\
- & nested $\mathbf{n}_{\mathrm{MVAB}}$ \\
- & $\mathbf{n}_{2 \mathrm{D}}$ on $\mathrm{C} 2$ \\
- & $\mathbf{n}_{2 \mathrm{D}}$ on $\mathrm{C} 4$ \\
- & $\mathbf{n}_{2 \mathrm{D}}$ on $\mathrm{C} 3$ \\
- & $\mathbf{n}_{2 \mathrm{D}}$ on $\mathrm{C} 1$
\end{tabular}

Fig. B1. Comparison between the C2_OpTA_A solution and the results from planar MVAB technique. Left column: The first four panels present the magnetic field variance (solid lines) and average normal magnetic field component (dashed lines) corresponding to MP intervals centred on $T_{c i}$. The intervals half-width, in seconds from $\tau_{i}$, is shown on the abscissa. The plotted quantities were computed based on three sets of normals, as explained in the text. The bottom panel presents the global normal magnetic field variances. Right column: The relative orientation of the three sets of normals as a function of interval half-width. In all the panels, the vertical dashed line corresponds to the central intervals $\left[T_{c i}-\tau_{i}, T_{c i}+\tau_{i}\right]$, used in the timing analysis. More explanations are given in the text.

In case of C2 (panel 6) the effect is still important for the central interval of $\tau_{2}$ half-width, meaning that the corresponding MVAB results are spoiled by local, internal irregularities (magnetic islands, noise etc.). As a consequence, for a better interpretation of the results, in Table 3 and in Table 5 the global normal magnetic field variance based both on data from all four satellites (black numbers) as well as only on data from $\mathrm{C} 1, \mathrm{C} 3$ and $\mathrm{C} 4$ (smaller blue numbers) is shown.

As mentioned in Sect. 5.2, for $\mathrm{C} 1$ and $\mathrm{C} 3$, there is a systematic difference in orientation between the individual 
Table C1. The relative standard deviation of the MP thickness, radius of curvature, and average normal velocities at each spacecraft, arising from the timing information inaccuracy. Results corresponding to three solutions are presented, i.e. C2_OpTA_A, C2_TA, and P2_OpTA, for two sets of values of the parameters $\delta_{i}$, measuring the timing information inaccuracy (see text for more explanations).

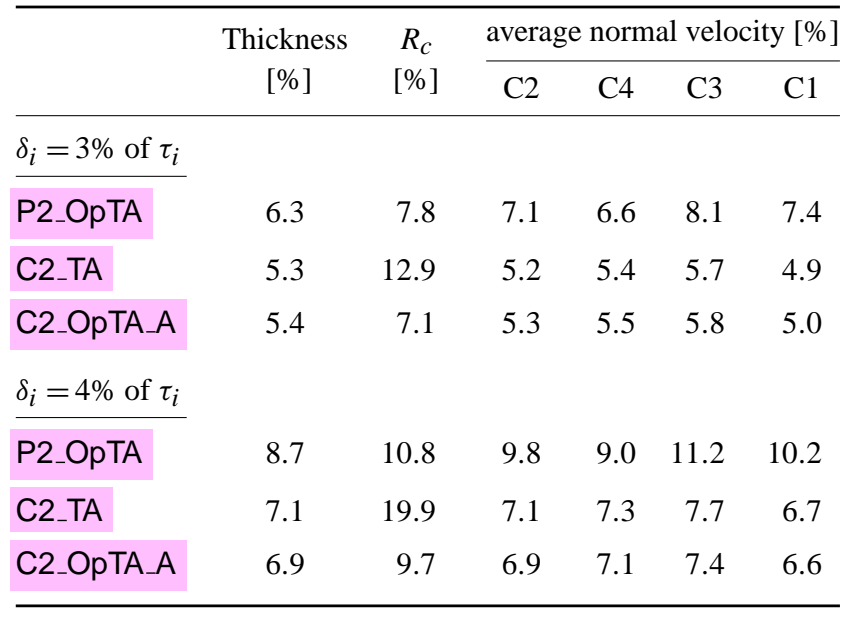

MVAB normals obtained in the nested analysis (called in this appendix the fixed, planar $M V A B$ normals) and the $\boldsymbol{n}_{2 \mathrm{D}}^{\text {ave nor- }}$ mals provided by all 2-D MP solutions. The panels 8 and 9 in Fig. B1 make clear that this difference in orientation is due to the different time-intervals on which the two normals are based, and is not an effect induced by the 2-D method. In fact, for C3 (panel 8) and C1 (panel 9) the planar, intervalspecific $M V A B$ normals are very close to the $\boldsymbol{n}_{2 \mathrm{D}}^{\text {ave }}$, when the interval half-width is $\tau_{3}$ and $\tau_{1}$, respectively. Moreover, for all the central intervals with the half-width greater than $\tau_{3}$, respectively $\tau_{1}$, the planar, interval-specific MVAB normals lie in the range of angular variation for the $2-D$, instantaneous normals, i.e. the orange line lies between the dashed, colour coded lines. This is exactly the result one expects when the planar MVAB technique is applied to an ideal 2-D discontinuity (see Dunlop and Woodward, 1998).

\section{Appendix C}

\section{Error calculation in the 2-D method}

Although the half-width crossing durations $\tau_{i}$ and the central crossing times $T_{c i}$ are obtained by minimizing the differences between the measured magnetic field and a model describing the MP large scale magnetic profile (see the beginning of Sect. 2.3), their values may be altered by the presence of local temporal/spatial variations. For the transition detected by Cluster on 24 June 2003, we estimated the errors in the values of MP crossing parameters arising from this timing information inaccuracy.
In the present appendix, the error calculation corresponding to the C2_OpTA_A, the C2_TA, and the P2_OpTA solutions will be presented for comparison. As discussed in Sect. 6, these solutions show similarities, with the first two being practically identical, and involve the highest dynamical behavior for the MP. One can therefore presume that their characteristics depend more on the timing information than in case of other solutions.

The error calculation was implemented by allowing $\tau_{i}$ and $T_{c i}, i=1 \ldots 4$, to take values within a certain range. Then, the 2-D method was applied for all possible combinations of the new $\tau_{i}$ and $T_{c i}$, and the relative standard deviation (RSD) of the MP crossing parameters was computed and associated with errors arising from timing information inaccuracy.

More precisely, we allowed $\tau_{i}$ and $T_{c i}, i=1 \ldots 4$, to vary independently within the limits $\tau_{i} \pm \delta_{i} / 2$ and $T_{c i} \pm \delta_{i} / 2$, respectively, with $\delta_{i}$ being a quantity specific to each satellite. After examining how different fitting curves of the type presented in Fig. 5, reproduce the general magnetic field evolution in the MP central part, we concluded that $\delta_{i}$ is of the order of $\sim 3-4 \% \tau_{i}$ for the analyzed event. The value $\delta_{i}=3 \% \tau_{i}$ (i.e. about $0.19 \mathrm{~s}$ for $\mathrm{C} 2,0.22 \mathrm{~s}$ for $\mathrm{C} 4,0.27 \mathrm{~s}$ for $\mathrm{C} 3$, and $0.28 \mathrm{~s}$ for $\mathrm{C} 1$ ), leads to a total variation in the times of MP edges encounter $T_{c i} \pm \tau_{i}$ (i.e. the input parameters in the new method) of around $0.39 \mathrm{~s}$ for $\mathrm{C} 2$ and $0.56 \mathrm{~s}$ for $\mathrm{C} 1$. When $\delta_{i}=4 \% \tau_{i}$ (i.e. about $0.26 \mathrm{~s}$ for $\mathrm{C} 2,0.29 \mathrm{~s}$ for $\mathrm{C} 4,0.36 \mathrm{~s}$ for $\mathrm{C} 3$, and $0.37 \mathrm{~s}$ for $\mathrm{C} 1$ ), the total variation in the times of $\mathrm{MP}$ edges encounter is around $0.52 \mathrm{~s}$ for $\mathrm{C} 2$ and $0.74 \mathrm{~s}$ for $\mathrm{C} 1$.

Table $\mathrm{C} 1$ presents the RSD of the MP macroscopic parameters, i.e. thickness, radius of curvature, and individual average normal velocities, in these two cases. It shows that the variations in the crossing parameters remain in a reasonably limit, with the most affected parameter being the radius of curvature. The three solutions that were analyzed allow to compare the influence of timing inaccuracy between the parabolic and cylindrical model, on one hand, and between the straight timing analysis and combined timing - MVAB analysis, on the other hand.

By comparing between the C2_TA and C2_OpTA_A solutions one can observe that the combined timing - MVAB analysis provides results significantly more stable with respect to timing inaccuracy for the MP radius of curvature, and similar or slightly more stable for the other MP macroscopic parameters. This effect is even more pronounced in case of the parabolic model, where the use of the optimized implementation proved to be crucial: as described at the beginning of Sect. 6, the solution P2_TA, provided by the straight timing analysis, had to be abandoned because it was unstable and does not offer a satisfactory physical description of the MP parameters and dynamics (see Blagau, 2007). As for the comparison between the parabolic and cylindrical model, the same remark and the results corresponding to the P2_OpTA and C2_OpTA_A solutions, indicate that the cylindrical model is less affected by timing inaccuracy. 
We tested the stability of the C2_OpTA_A solution in another way, namely by investigating how the MP macroscopic parameters change when the magnetic levels used to set the timing conditions are changed. The original timing information, extracted from the satellites detecting the $B_{m} \pm 1 / 2 \Delta B \tanh (1)$ magnetic levels (therefore corresponding to a fraction of $\tanh (1) \sim 76.2 \%$ from the total MP magnetic jump), was changed to correspond to other three sets of magnetic levels, that encompass symmetrically a fraction of $\tanh (1.1) \sim 80.0 \%, \tanh (1.2) \sim 83.4 \%$ and $\tanh (1.3) \sim 86.2 \%$ from the total MP magnetic change. Stable values for the MP macroscopic parameters have been found, with the radius of curvature varying by $\sim 4.0 \%, \sim$ $7.1 \%$, and $\sim 8.4 \%$, respectively. The average normal velocity and the MP thickness changed by $\sim 3.2 \%, \sim 6.8 \%$, and $\sim 10.3 \%$, respectively.

Acknowledgements. We acknowledge the efforts of the FGM instrument team (Principal Investigator, Elizabeth Lucek from Imperial College, London), the CIS team, (Principal Investigators Henri Rème and Iannis Dandouras from Centre d'Etude Spatiale des Rayonnements, Toulouse), and the EFW team (Principal Investigator, Mats André from Swedish Institute of Space Physics, Uppsala) for the effort of building and operating their instruments.

The authors thank Per-Arne Lindqvist and Tomas Karlsson from the Alfvén Laboratory, Stockholm, and Bjørn Lybekk and Arne Dahlback from the University of Oslo, for providing the EFW data.

The service charges for this open access publication have been covered by the Max Planck Society.

Topical Editor I. A. Daglis thanks A. Vaivads and another anonymous referee for their help in evaluating this paper.

\section{References}

Balogh, A., Carr, C. M., Acuña, M. H., Dunlop, M. W., Beek, T. J., Brown, P., Fornacon, K.-H., Georgescu, E., Glassmeier, K.H., Harris, J., Musmann, G., Oddy, T., and Schwingenschuh, K.: The Cluster Magnetic Field Investigation: overview of in-flight performance and initial results, Ann. Geophys., 19, 1207-1217, 2001 , http://www.ann-geophys.net/19/1207/2001/.

Blagau, A.: Characteristics of Earth's magnetopause from Cluster measurements, PhD thesis, Max-Planck-Institut für extraterrestrische Physik, Garching, Germany, http://edoc.ub. uni-muenchen.de/7806/, 2007.

Chandrasekhar, S.: Hydrodynamic and hydromagnetic stability, International Series of Monographs on Physics, Clarendon, Oxford, 1961.

de Keyser, J.: Empirical Reconstruction, in: Multi-Spacecraft Analysis Methods Revisited, edited by: Paschmann, G. and Daly, P. W., ISSI Scientific Reports SR-008, pp. 91-97, ESA Publications Division, 2008.

de Keyser, J. and Roth, M.: Structural analysis of periodic surface waves on the magnetospheric boundary, Planet. Space Sci., 51, 757-768, doi:10.1016/S0032-0633(03)00112-0, 2003.
Dunlop, M. W. and Woodward, T. I.: Multi-Spacecraft Discontinuity Analysis: Orientation and Motion, in: Analysis Methods for Multi-Spacecraft Data, edited by: Paschmann, G. and Daly, P. W., ISSI Scientific Reports SR-001, pp. 271-306, ESA Publications Division, 1998.

Dunlop, M. W. and Woodward, T. I.: Analysis of thick, non-planar boundaries using the discontinuity analyser, Ann. Geophys., 17, 984-995, 1999, http://www.ann-geophys.net/17/984/1999/.

Dunlop, M. W., Balogh, A., Cargill, P., Elphic, R. C., Fornaon, K.-H., Georgescu, E., Sedgemore-Schulthess, F., and the FGM team: Cluster observes the Earth's magnetopause: coordinated four-point magnetic field measurements, Ann. Geophys., 19, 1449-1460, 2001, http://www.ann-geophys.net/19/1449/2001/.

Dunlop, M. W., Balogh, A., and Glassmeier, K.-H.: Four-point Cluster application of magnetic field analysis tools: The discontinuity analyzer, J. Geophys. Res., 107, 1385-1397, doi: 10.1029/2001JA005089, 2002.

Foullon, C., Farrugia, C. J., Fazakerley, A. N., Owen, C. J., Gratton, F. T., and Torbert, R. B.: Evolution of Kelvin-Helmholtz activity on the dusk flank magnetopause, J. Geophys. Res., 113, 1120311224, doi:10.1029/2008JA013175, 2008.

Gustafsson, G., André, M., Carozzi, T., Eriksson, A. I., Fälthammar, C.-G., Grard, R., Holmgren, G., Holtet, J. A., Ivchenko, N., Karlsson, T., Khotyaintsev, Y., Klimov, S., Laakso, H., Lindqvist, P.-A., Lybekk, B., Marklund, G., Mozer, F., Mursula, K., Pedersen, A., Popielawska, B., Savin, S., Stasiewicz, K., Tanskanen, P., Vaivads, A., and Wahlund, J.-E.: First results of electric field and density observations by Cluster EFW based on initial months of operation, Ann. Geophys., 19, 1219-1240, 2001 , http://www.ann-geophys.net/19/1219/2001/.

Haaland, S. E., Sonnerup, B. U. Ö., Dunlop, M. W., Balogh, A., Georgescu, E., Hasegawa, H., Klecker, B., Paschmann, G., PuhlQuinn, P., Rème, H., Vaith, H., and Vaivads, A.: Four-spacecraft determination of magnetopause orientation, motion and thickness: comparison with results from single-spacecraft methods, Ann. Geophys., 22, 1347-1365, 2004, http://www.ann-geophys.net/22/1347/2004/.

Hasegawa, H., Sonnerup, B. U. Ö., Klecker, B., Paschmann, G., Dunlop, M. W., and Rème, H.: Optimal reconstruction of magnetopause structures from Cluster data, Ann. Geophys., 23, 973982, 2005,

http://www.ann-geophys.net/23/973/2005/.

Khrabrov, A. and Sonnerup, B. U. Ö.: deHoffmann-Teller Analysis, in: Analysis Methods for Multi-Spacecraft Data, edited by: Paschmann, G. and Daly, P. W., ISSI Scientific Reports SR-001, pp. 221-248, ESA Publications Division, 1998a.

Khrabrov, A. V. and Sonnerup, B. U. Ö.: Orientation and motion of current layers: Minimization of the Faraday residue, Geophys. Res. Lett., 25, 2373-2376, doi:10.1029/98GL51784, 1998b.

Kivelson, M. G. and Chen, S.-H.: The Magnetopause: Surface Waves and Instabilities and their Possible Dynamical Consequences, in: Physics of the Magnetopause, edited by: Song, P., Sonnerup, B. U. Ö., and Thomsen, M. F., vol. 90 of Geophysical Monograph, pp. 257-268, American Geophysical Union, Washington, USA, 1995.

Mottez, F. and Chanteur, G.: Surface crossing by a group of satel- 
lites: A theoretical study, J. Geophys. Res., 99, 13499-13507, doi:10.1029/93JA03326, 1994.

Owen, C. J., Taylor, M. G. G. T., Krauklis, I. C., Fazakerley, A. N., Dunlop, M. W., and Bosqued, J. M.: Cluster observations of surface waves on the dawn flank magnetopause, Ann. Geophys., 22, 971-983, 2004,

http://www.ann-geophys.net/22/971/2004/.

Paschmann, G. and Sonnerup, B. U. Ö.: Proper Frame Determination and Walén Test, in: Multi-Spacecraft Analysis Methods Revisited, edited by: Paschmann, G. and Daly, P. W., ISSI Scientific Reports SR-008, pp. 65-74, ESA Publications Division, 2008.

Rème, H., Aoustin, C., Bosqued, J. M., Dandouras, I., Lavraud, B., Sauvaud, J. A., Barthe, A., Bouyssou, J., Camus, Th., CoeurJoly, O., Cros, A., Cuvilo, J., Ducay, F., Garbarowitz, Y., Medale, J. L., Penou, E., Perrier, H., Romefort, D., Rouzaud, J., Vallat, C., Alcaydé, D., Jacquey, C., Mazelle, C., d'Uston, C., Möbius, E., Kistler, L. M., Crocker, K., Granoff, M., Mouikis, C., Popecki, M., Vosbury, M., Klecker, B., Hovestadt, D., Kucharek, H., Kuenneth, E., Paschmann, G., Scholer, M., Sckopke, N., Seidenschwang, E., Carlson, C. W., Curtis, D. W., Ingraham, C., Lin, R. P., McFadden, J. P., Parks, G. K., Phan, T., Formisano, V., Amata, E., Bavassano-Cattaneo, M. B., Baldetti, P., Bruno, R., Chionchio, G., Di Lellis, A., Marcucci, M. F., Pallocchia, G., Korth, A., Daly, P. W., Graeve, B., Rosenbauer, H., Vasyliunas, V., McCarthy, M., Wilber, M., Eliasson, L., Lundin, R., Olsen, S., Shelley, E. G., Fuselier, S., Ghielmetti, A. G., Lennartsson, W., Escoubet, C. P., Balsiger, H., Friedel, R., Cao, J.-B., Kovrazhkin, R. A., Papamastorakis, I., Pellat, R., Scudder, J., and Sonnerup, B.: First multispacecraft ion measurements in and near the Earth's magnetosphere with the identical Cluster ion spectrometry (CIS) experiment, Ann. Geophys., 19, 1303-1354, 2001, http://www.ann-geophys.net/19/1303/2001/.
Roelof, E. C. and Sibeck, D. G.: Magnetopause shape as a bivariate function of interplanetary magnetic field $B_{z}$ and solar wind dynamic pressure, J. Geophys. Res., 98, 21421-21450, doi: 10.1029/93JA02362, 1993.

Russell, C. T., Mellott, M. M., Smith, E. J., and King, J. H.: Multiple spacecraft observations of interplanetary shocks Four spacecraft determination of shock normals, J. Geophys. Res., 88, 4739-4748, doi:10.1029/JA088iA06p04739, 1983.

Sonnerup, B. U. O. and Cahill, Jr., L. J.: Magnetopause Structure and Attitude from Explorer 12 Observations, J. Geophys. Res., 72, 171-183, doi:10.1029/JZ072i001p00171, 1967.

Sonnerup, B. U. Ö. and Scheible, M.: Minimum and maximum variance analysis, in: Analysis Methods for Multi-Spacecraft Data, edited by: Paschmann, G. and Daly, P. W., ISSI Scientific Reports SR-001, pp. 185-220, ESA Publications Division, 1998.

Sonnerup, B. U. O., Papamastorakis, I., Paschmann, G., and Luehr, H.: Magnetopause properties from AMPTE/IRM observations of the convection electric field - Method development, J. Geophys. Res., 92, 12137-12159, doi:10.1029/JA092iA11p12137, 1987.

Sonnerup, B. U. Ö., Haaland, S., Paschmann, G., Dunlop, M. W., Rème, H., and Balogh, A.: Orientation and motion of a plasma discontinuity from single-spacecraft measurements: Generic residue analysis of Cluster data, J. Geophys. Res., 111, 52035218, doi:10.1029/2005JA011538, 2006.

Sonnerup, B. U. Ö., Teh, W.-L., and Hasegawa, H.: Grad-Shafranov and MHD Reconstructions, in: Multi-Spacecraft Analysis Methods Revisited, edited by: Paschmann, G. and Daly, P. W., ISSI Scientific Reports SR-008, pp. 81-90, ESA Publications Division, 2008. 